\title{
Raman Spectroscopy and Imaging of Graphene
}

\author{
Zhenhua Ni, Yingying Wang, Ting Yu, and Zexiang Shen ( $\bowtie)$ \\ Division of Physics and Applied Physics, School of Physical and Mathematical Sciences, Nanyang Technological University, Singapore \\ 637371, Singapore \\ Received: 17 July 2008/ Revised: 27 August 2008/Accepted: 28 August 2008 \\ CTsinghua Press and Springer-Verlag 2008. This article is published with open access at Springerlink.com
}

\begin{abstract}
Graphene has many unique properties that make it an ideal material for fundamental studies as well as for potential applications. Here we review recent results on the Raman spectroscopy and imaging of graphene. We show that Raman spectroscopy and imaging can be used as a quick and unambiguous method to determine the number of graphene layers. The strong Raman signal of single layer graphene compared to graphite is explained by an interference enhancement model. We have also studied the effect of substrates, the top layer deposition, the annealing process, as well as folding (stacking order) on the physical and electronic properties of graphene. Finally, Raman spectroscopy of epitaxial graphene grown on a SiC substrate is presented and strong compressive strain on epitaxial graphene is observed. The results presented here are highly relevant to the application of graphene in nano-electronic devices and help in developing a better understanding of the physical and electronic properties of graphene.
\end{abstract}

\section{KEYWORDS}

Graphene, Raman spectroscopy and imaging, substrate effect, device application

\section{Introduction}

Graphene comprises one monolayer of carbon atoms packed into a two-dimensional (2D) honeycomb lattice [1]. It has attracted much interest since it was first discovered in 2004 [2]. Graphene is the basic building block for other carbon nanomaterials, such as $0 \mathrm{D}$ fullerenes, 1D carbon nanotubes, and 2D nanographite sheets. In the electronic band structure of graphene, the conduction band touches the valence band at two points $\left(K\right.$ and $\left.K^{\prime}\right)$ in the Brillouin zone, and in the vicinity of these points, the electron energy has a linear relationship with the wavevector, $E=$ $\hbar k v_{\mathrm{f}}$. Therefore, electrons in an ideal graphene sheet behave like massless Dirac-Fermions [1]. The unique properties of graphene make it a promising candidate for fundamental study as well as for potential device applications [3-8]. The charge carriers in graphene can be tuned continuously between electrons and holes in concentrations $n$ as high as $10^{13} / \mathrm{cm}^{2}$. The mobility of graphene at room temperature is $\sim 120000$ $\mathrm{cm}^{2} /(\mathrm{V} \cdot \mathrm{s})$, higher than any known semiconductor $[9,10]$. In addition, the application of graphene in spintronics has also received a lot of interest [11, 12]. A spin relaxation length of $\sim 2 \mu \mathrm{m}$ has been observed in graphene, which suggests a promising future for graphene spintronics. Many other applications of graphene have also been reported [5, 12-15].

Address correspondence to zexiang@ntu.edu.sg

\section{算 Springer}


Raman spectroscopy has historically been used to probe structural and electronic characteristics of graphite materials, providing useful information on the defects ( $\mathrm{D}$ band), in-plane vibration of $\mathrm{sp}^{2}$ carbon atoms ( $\mathrm{G}$ band) as well as the stacking order (2D band) [16-18]. Raman spectroscopic studies of graphene have revealed very interesting phenomena. For example, the single and sharp second order Raman band (2D) has been widely used as a simple and efficient way to confirm the presence of single layer graphene (SLG) [19]. The 2D band of multilayer graphene can be fitted with multiple peaks, due to the splitting of the electronic band structure of the multilayer material [20]. The electronic structure of bilayer graphene (BLG) has also been probed by resonant Raman scattering [21]. Electrical field effect studies have revealed that electron/hole doping in graphene will affect the electron-phonon coupling, and hence the Raman frequency [22-24]. The inplane vibrational $G$ band of graphene is blueshifted for both electron and hole doping, due to the nonadiabatic removal of the Kohn anomaly at the $\Gamma$ point. On the other hand, the $2 \mathrm{D}$ band is redshifted for electron doping and blueshifted for hole doping, due to the charge transfer induced modification of the equilibrium lattice parameter [25]. This makes Raman spectroscopy an effective technique to determine the doping type and dopant concentration in graphene $[26,27]$. The effects of temperature on the Raman spectra of graphene have also been studied and the lattice inharmonicity of graphene investigated [28]. Moreover, an extremely high thermal conductivity of graphene $(\sim 3080-5150 \mathrm{~W} /(\mathrm{m} \cdot \mathrm{K}))$ has recently been observed by Raman spectroscopy, which suggests the potential application of graphene as a thermal management material in future nanoelectronic devices [29].

In this contribution, Raman spectroscopy and imaging are used to study several aspects of graphene structure: (i) Raman spectroscopy and imaging are used as an efficient way to determine the number of graphene layers; (ii) The strong signal of graphene on a $\mathrm{SiO}_{2} / \mathrm{Si}$ substrate is explained by an interference enhancement model; (iii) The effect of substrates, top insulator deposition and annealing processes, as well as folding and stacking order on the physical and electronic structure of graphene are studied by Raman spectroscopy and imaging; (iv) Raman studies of epitaxial graphene grown on an $\mathrm{SiC}$ substrate are also presented.

\section{Preparation of graphene}

Several approaches have been successfully developed to fabricate graphene, such as micromechanical cleavage of graphite [2] and epitaxial growth on $\mathrm{SiC}$ substrates [30,31]. The former can be used to obtain high quality graphene sheets which are comparable to that in graphite, but is restricted by small sample dimensions and low visibility. Epitaxial graphene (EG) grown on $\mathrm{SiC}$ is suitable for large area fabrication and is more compatible with current Si processing techniques for future applications. However, the quality of EG still needs to be improved and the effects of the substrate on its properties are not well understood. In addition to the above methods, a number of studies have also reported the growth of graphene on metal substrates, such as ruthenium [32] and nickel [33], but these methods require the sample to be transferred to insulating substrates in order to make useful devices. There are also liquid phase exfoliation approaches to make graphene sheet films by dispersion and exfoliation of graphite in organic solvents $[13,34]$. Chemical routes have also been developed to fabricate graphene and graphene nanostructures and interesting results reported $[35,36]$. In the work described in this paper, the samples were prepared by micromechanical cleavage or epitaxial growth on $\mathrm{SiC}$ substrates.

\section{Raman spectroscopy and imaging of graphene and layer thickness determination}

A quick and precise method for determining the number of layers of graphene sheets is essential to accelerate research and exploitation of graphene. Although atomic force microscopy (AFM) measurement is the most direct way to identify the number of layers of graphene, the method has a very slow throughput. Furthermore, an instrumental offset of $\sim 0.5 \mathrm{~nm}$ (caused by different interaction forces) always exists, which is even larger than the thickness 
of a graphene monolayer and data fitting is required to extract the true thickness of graphene sheets [37]. Unconventional quantum Hall effects $[3,38]$ are often used to differentiate monolayer and bilayer graphene from few layer materials. However, this is not a practical or efficient method. Researchers have attempted to develop more efficient ways to identify different numbers of layers of graphene without destroying the crystal lattice. Raman spectroscopy has been shown to be a potential candidate for nondestructive and quick characterization of the number of layers of graphene [19, 39].

Figure 1 gives typical Raman spectra of SLG and graphite on a $\mathrm{SiO}_{2}(300 \mathrm{~nm}) / \mathrm{Si}$ substrate. The Raman spectra were recorded with a WITec CRM200 Raman system with a double-frequency Nd:YAG laser (532 nm) as excitation source. The laser power at the sample is below $0.1 \mathrm{~mW}$ in order to avoid laserinduced heating. The two spectra were recorded under the same experimental conditions. The Raman signal of SLG is unexpectedly strong and even comparable to that of bulk graphite. This interesting phenomenon will be addressed in Section 3 below [40]. The major Raman features of graphene and graphite are the so called G band $\left(\sim 1580 \mathrm{~cm}^{-1}\right)$ and 2D band $\left(\sim 2670 \mathrm{~cm}^{-1}\right)$. The $\mathrm{G}$ band originates from in-plane vibration of $\mathrm{sp}^{2}$ carbon atoms and is a doubly degenerate (TO and LO) phonon mode $\left(E_{2 g}\right.$ symmetry) at the Brillouin zone center [41]. The 2D band originates from a two phonon double resonance

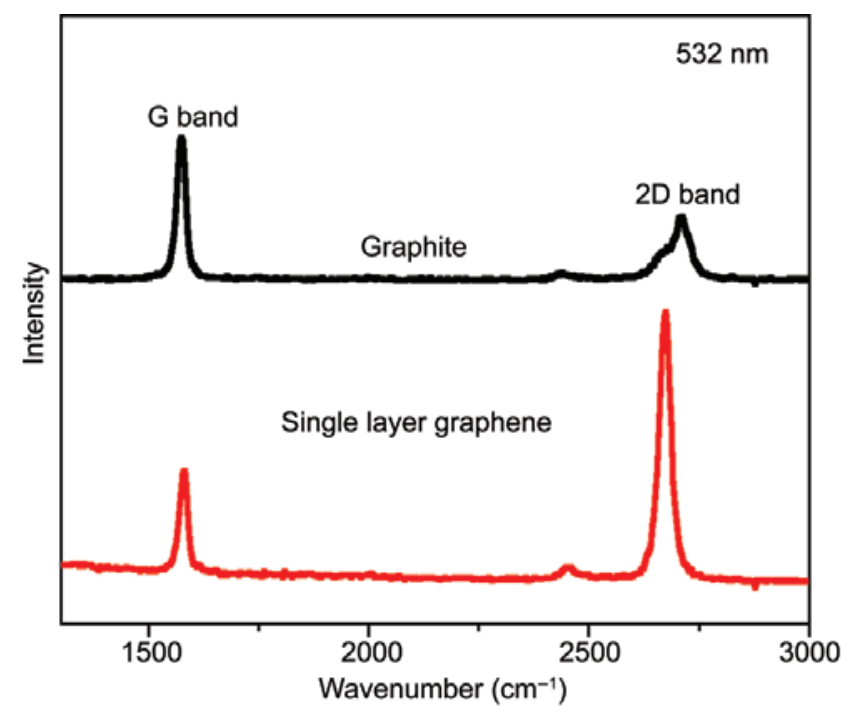

Figure 1 Raman spectra of single layer graphene and graphite
Raman process [42]. The 2D band is also denoted the $G^{\prime}$ band in some work [20], as it is the second most prominent band of graphite samples after the $G$ band. Here, we refer to it as the $2 \mathrm{D}$ band to highlight the fact that it is the second order overtone of the $\mathrm{D}$ band. The obvious difference between the Raman features of SLG and graphite is the 2D band. For SLG, the 2D band can be fitted with a sharp and symmetric peak while that of graphite can be fitted with two peaks. It can be seen in Fig. 2 that the 2D band becomes broader and blueshifted when the graphene thickness increases from SLG to multilayer graphene. As the 2D band originates from a two phonon double resonance process, it is closely related to the band structure of graphene layers. Ferrari et al. [19] have successfully used the splitting of the electronic band structure of multilayer graphene to explain the broadening of the $2 \mathrm{D}$ band. Cancado et al. [20] also gave a detailed description of how the multipeak structure of the $2 \mathrm{D}$ band observed for a stacking of two or more graphene layers is related to the dispersion of $\pi$ electrons. As a result, the presence of a sharp and symmetric 2D band is widely used to identify SLG [5]. Unfortunately, the differences in the 2D band between two and few layers of graphene sheets are not unambiguous in the Raman spectra. In addition to the differences in the $2 \mathrm{D}$ band, the intensity of the $\mathrm{G}$ band increases almost linearly as the graphene thickness increases, as shown in Fig. 2 [40]. This can be readily understood as more carbon atoms are detected for multi-layer graphene. Therefore, the intensity of the $G$ band can be used to determine the number of layers of graphene.

Figure 3(a) shows the optical image of a graphene sample on the $\mathrm{SiO}_{2} / \mathrm{Si}$ substrate. The graphene sheet contains one, two, and three layers, as predetermined by AFM. Figure 3(b) shows the Raman spectra of graphene with different thicknesses, as well as that of highly ordered pyrolytic graphite (HOPG) for comparison. Figure 3(c) shows the Raman imaging of the $G$ band intensity of the graphene sheets. The differences between graphene samples with different thicknesses are very obvious. The intensity of the $G$ band along the dashed line is shown in Fig. 3(d). It can be clearly seen that the intensity of the $G$ band increases almost linearly

\section{圈国 Springer}




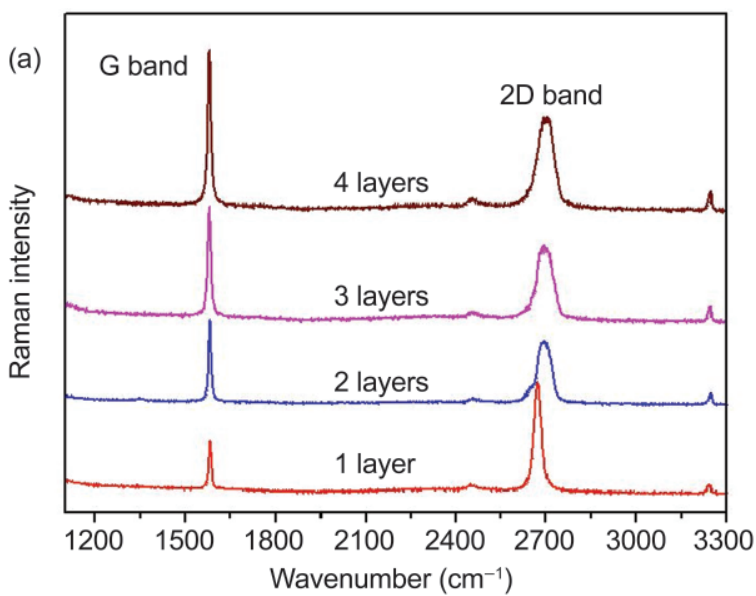

the contrast spectroscopy can also be used to accurately identify the graphene layer numbers (up to 10 layers), which offers a cheaper alternative [44]. The contrast between graphene sheets and the substrate is generated and quantified in a contrast spectrum by the following equation:

$$
C(\lambda)=\frac{R_{0}(\lambda)-R(\lambda)}{R_{0}(\lambda)}
$$

By using the contrast method, the number of layers in a graphene sheet on a $300 \mathrm{~nm} \mathrm{SiO}_{2} / \mathrm{Si}$ substrate can be determined precisely [44] by using the following equation:

$$
C=0.0046+0.0925 N-0.00255 N^{2}
$$

with the increase in graphene thickness. This can be used to determine the number of layers of multilayer graphene. The advantage of Raman spectroscopy and imaging in determining the number of graphene layers is that it does not depend on the substrate used. This is because the Raman spectrum is an intrinsic characteristic of graphene.

In addition to the Raman spectroscopy/imaging, where $N(\leq 10)$ is the number of layers of graphene.

Although current research mainly focuses on single and bilayer graphene, we believe that few layer (less than 10 layers) graphene materials also have interesting properties as they still exhibit twodimensional properties [1]. By combining Raman spectroscopy and the contrast method, the number of
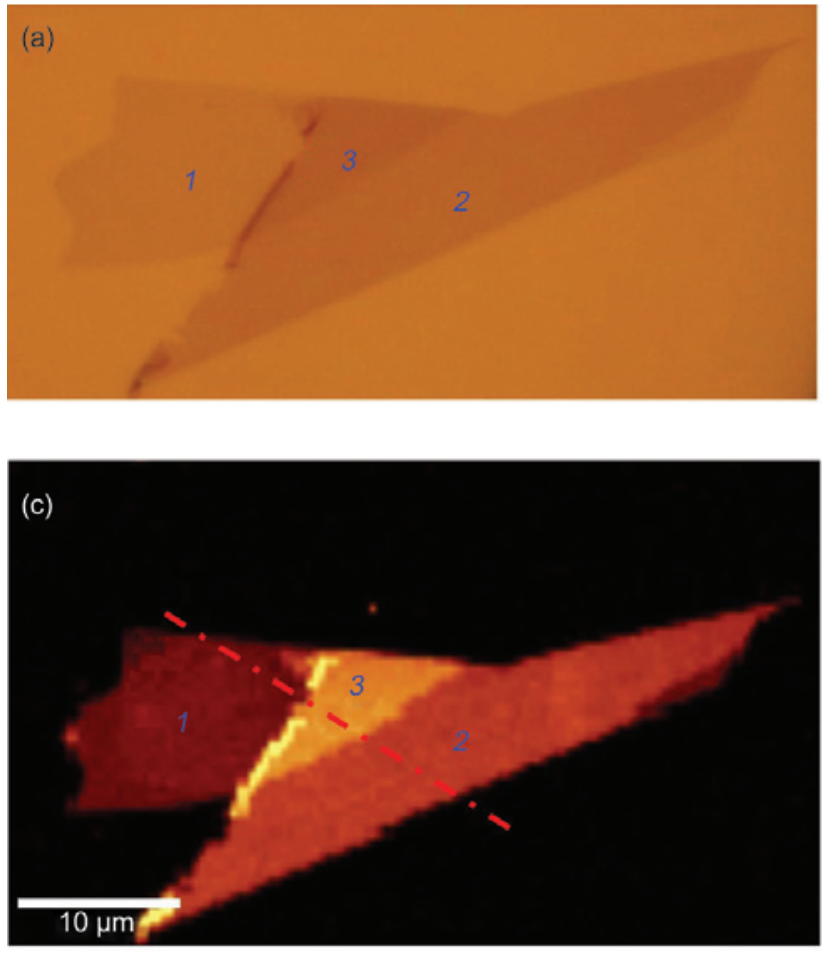
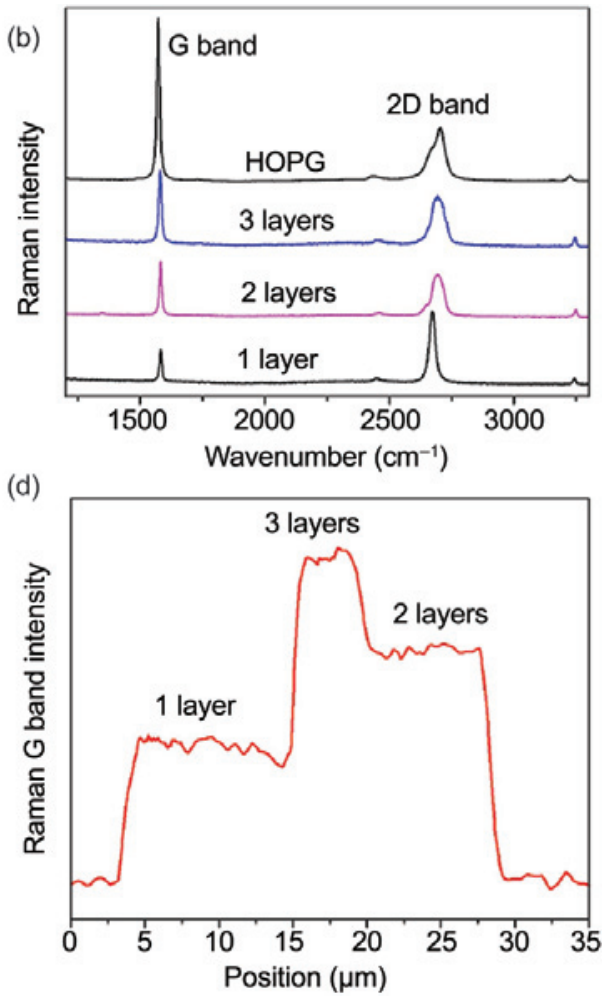

Figure 3 (a) Optical image of graphene with 1, 2, and 3 layers. (b) Raman spectra of graphene with 1, 2, and 3 layers as well as that of HOPG. (c) Raman image plotted by intensity of the G band. (d) The intensity cross-section of the Raman image corresponding to the dashed line in (c) 
graphene layers can be unambiguously identified in any substrate, which stimulates future research and applications of graphene.

\section{Interference enhancement of graphene on a $\mathrm{SiO}_{2} / \mathrm{Si}$ substrate}

The very strong Raman signal from graphene (single and few layer) on $\mathrm{SiO}_{2} / \mathrm{Si}$ substrates has been observed by many researchers [19, 37, 39]. Normally, the Raman intensity is expected to be proportional to the thickness of the graphene sample up to the laser penetration depth, which is around $50 \mathrm{~nm}$ ( 150 layers) for $532 \mathrm{~nm}$ excitation. The penetration depth $z$ of a $532 \mathrm{~nm}$ laser in graphite is estimated by $\frac{I}{I_{0}}=\mathrm{e}^{-a z}=\frac{1}{\mathrm{e}}$, where $\alpha=\frac{4 \pi k}{\lambda}, \lambda=532 \mathrm{~nm}$, and $k=1.3$ is the extinction coefficient of graphite [45]. However, we find that the intensity of the G band Raman signal of graphene sheets decreases when the number of layers of graphene exceeds a certain value. Figure 4 shows the $\mathrm{G}$ band Raman intensity of graphene sheets on a $300 \mathrm{~nm} \mathrm{SiO}{ }_{2} / \mathrm{Si}$ substrate as a function of the number of layers [40]. The number of layers of graphene sheets is determined by Raman and contrast spectroscopy [44, 46, 47]. In addition to the graphene sheets with one, two, three, five, six, eight, and nine layers, samples $a-h$ have more than 10 layers, with thickness in the range $\sim 5 \mathrm{~nm}$ (sample $a$ ) to $\sim 50 \mathrm{~nm}$ (sample $h$ ), as determined by

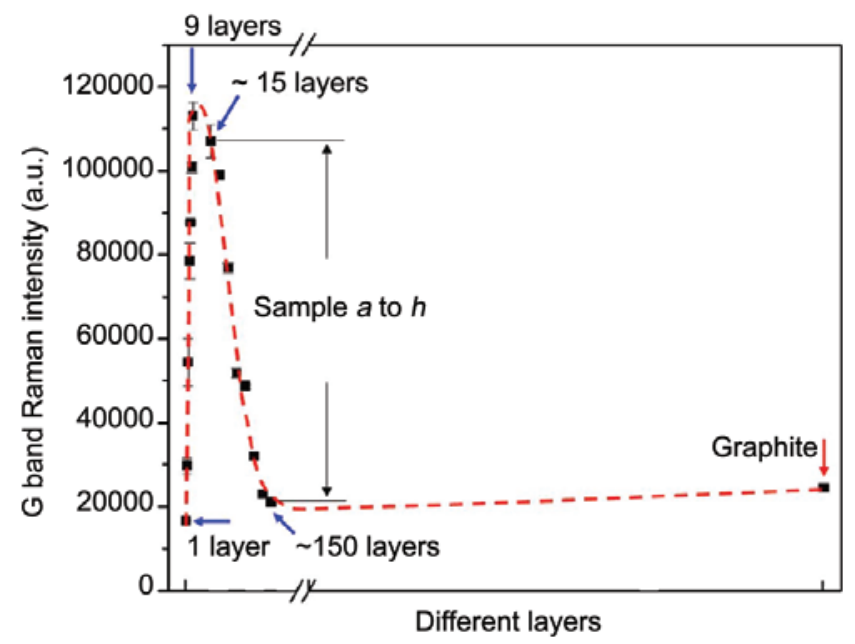

Figure 4 The $\mathrm{G}$ band Raman intensity of graphene sheets as a function of the number of layers. The red dashed curve is a guide to the eye. Reproduced with permission from Appl. Phys. Lett. 2008, 92, 043121. Copyright 2008 American Institute of Physics [40]
AFM. Interestingly, it can be seen that the Raman intensity increases almost linearly until $\sim 10$ layers $(\sim 3 \mathrm{~nm})$ and then decreases for thicker graphite sheets. Furthermore, the Raman signal of bulk graphite is obviously weaker than that of BLG. These results deviate from the above suggestion that laser penetration depths determine the thickness which gives the highest Raman intensity. Therefore, an alternative mechanism must be responsible for this phenomenon.

Multi-reflection/interference effects on contrast have been well documented in the literature but the multiple reflection of Raman signals has not [44, 46 , 47]. By considering the multilayer interference of incident light as well as the multi-reflection of Raman signals in graphene/graphite based on Fresnel's equations, the above Raman results can be explained. Consider the incident light passing from air onto a graphene sheet $/ \mathrm{SiO}_{2} / \mathrm{Si}$ trilayer system, as shown in Fig. 5(a). When a light front meets an interface, for example, the air/graphene or graphene $/ \mathrm{SiO}_{2}$ interface, a portion of the beam is reflected and the rest is transmitted, and thus, an infinite number of optical paths is possible. In Fig.

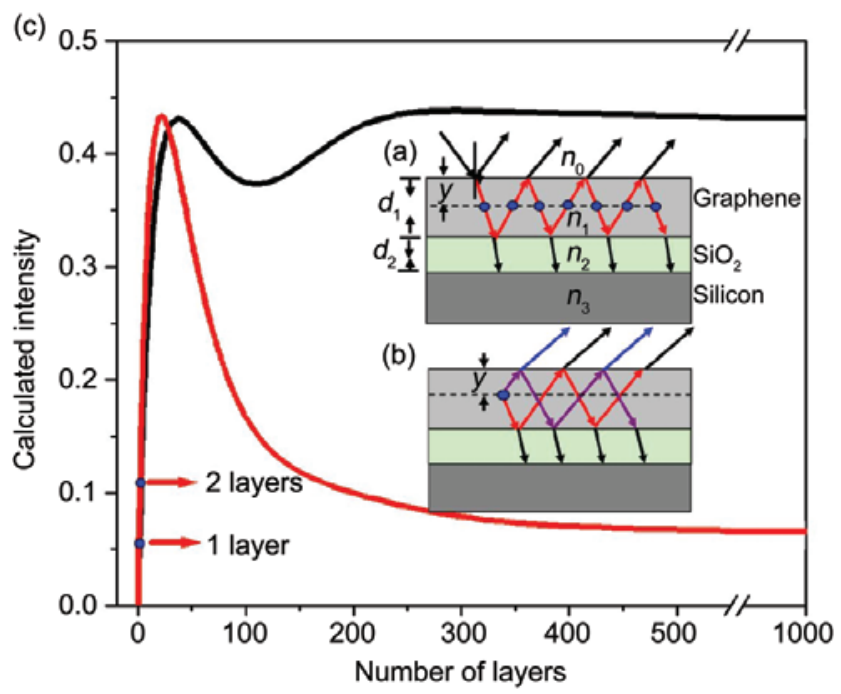

Figure 5 (a) Schematic laser reflection and transmission at a certain depth $y$ in graphene sheets deposited on a $\mathrm{SiO}_{2} / \mathrm{Si}$ substrate. (b) Multi-reflection of the scattering Raman light (from depth $y$ ) at the interfaces graphene/air and graphene/ $/ \mathrm{SiO}_{2}$. (c). Calculated results of Raman $\mathrm{G}$ band intensity as a function of the number of layers with (red) and without (black) consideration of the multi-reflection of Raman scattering light in graphene. Reproduced with permission from Appl. Phys. Lett. 2008, 92, 043121. Copyright 2008 American Institute of Physics [40] 
$5(\mathrm{a}), n_{0}=1$ is the refractive index of air, $n_{1}=2.6-1.3 \mathrm{i}$, $n_{2}=1.46, n_{3}=4.15-0.044 \mathrm{i}$, are the refractive indices of graphite, $\mathrm{SiO}_{2}$, and $\mathrm{Si}$ at $532 \mathrm{~nm}$, respectively [48], $d_{1}$ is the thickness of graphene which is estimated as $d_{1}=N \Delta d$, where $\Delta d=0.335 \mathrm{~nm}$ is the thickness of single layer graphene and $N$ is the number of layers, and $d_{2}$ is the thickness of $\mathrm{SiO}_{2}$ and the $\mathrm{Si}$ substrate is considered as semi-infinite. The $\mathrm{G}$ band Raman intensity of graphene sheets depends on the electric field distribution, which is a result of interference between all transmitted optical paths in graphene/graphite sheets. The total amplitude of the electric field at a certain depth $y$ in graphene/ graphite sheets is viewed as an infinite sum of the transmitted laser intensities, as schematically shown in Fig. 5(a). In addition, further consideration should be given to the multi-reflection of scattering Raman light in graphene/graphite at the graphene/air and graphene $/ \mathrm{SiO}_{2}$ interfaces, which contribute to the detected Raman signal. Figure 5(b) shows schematically the multi-reflection of the scattering light at a certain depth $y$ in graphene sheets. Thus, the total Raman signal is a result of interference of transmitted laser light followed by multi-reflection of Raman scattering light [40].

Figure 5(c) shows the calculated results of Raman $G$ band intensity as a function of the number of graphene/graphite layers. The black curve shows the calculated result without considering the multireflection of Raman light in graphene. It can be seen that the Raman scattering intensity is strongest at 38 layers and the scattering intensity of bulk graphite ( 1000 layers) is much stronger than that of single and bilayer graphene, which is not consistent with the experimental results. The red curve gives the calculated results after considering the multireflection of Raman light in graphene. In this case, the intensity is strongest at $\sim 22$ layers and the Raman signal of bulk graphite is weaker than that of BLG, which agrees very well with the experimental data. Thus, the consideration of the multi-reflection of scattering Raman light in graphene/graphite is clearly necessary. Our calculations of the Raman intensity of graphene can also be applied to other thin films and materials.

The idea of interference enhancement can also be applied to other thin film samples in order to enhance the Raman signal. A general selection of capping layers and substrates for enhancement can be achieved by choosing $n_{1}>>n_{2}<<n_{3}$, plus $n_{2} d_{2}=\frac{\lambda}{4}$, $\frac{3 \lambda}{4}$, and so on (where $n_{1}, n_{2}, n_{3}$ are the refractive indices of the sample, capping layer and substrate, respectively). A total phase change of $2 \pi$ (a change of $\pi$ at the capping layer/substrate interface, plus a change of $\pi$ due to the double thickness of the capping layer) can be achieved by this kind of configuration. Therefore, all the transmitted light in the graphene layers will have no phase change and thus will greatly enhance the Raman signal.

\section{Raman studies of single layer graphene: the substrate effect}

In the following Sections (4-6), we will individually discuss the effect of substrates, top layer deposition, annealing process, as well as folding on the atomic and electronic properties of graphene as probed by Raman spectroscopy and imaging.

To date, most Raman studies have been carried out on graphene sheets fabricated by micromechanical cleavage $(\mathrm{MC})$ and transferred to Si substrates with an appropriate thickness of a $\mathrm{SiO}_{2}$ capping layer ( 300 nm) [19, 39, 44, 47]. However, specific substrates might be required for different applications, such as transparent substrates for optical applications. A clear understanding of the interaction between graphene and a substrate is important for the development of potential applications of graphene and device fabrication. A systematic Raman study of single layer graphene (SLG) produced by MC on different substrates is presented here [43]. The choice of SLG is based on several factors. Firstly, it can be identified unambiguously by Raman spectroscopy from its characteristic 2D band feature. Secondly, compared with few layer graphene, SLG is more sensitive to the interactions between graphene sheets and substrate. We also compared the Raman features of SLG on different substrates with those of epitaxial monolayer graphene (EMG) grown on a $\mathrm{SiC}$ substrate, for which we believe there is a much stronger interaction between graphene and substrate. 
SLG was fabricated on different substrates, such as transparent materials (glass and quartz), flexible materials (polyethylene terephthalate (PET) and polydimethylsiloxane (PDMS)), conductive materials (NiFe and highly doped $\mathrm{Si}$ ), as well as the standard $\mathrm{SiO}_{2} / \mathrm{Si}$ substrate. These substrates are suitable for a variety of applications. Figure 6 shows the Raman spectra of SLG on the different substrates, as well as the Raman spectrum of EMG grown on a $\mathrm{SiC}$ substrate. The SLG graphene was identified by both the sharp 2D Raman band and the contrast method [43]. The positions of the $\mathrm{G}$ band and 2D band and their full width at half maximum (FWHM) are summarized in Table 1 for the different substrates. The results for graphene on a GaAs substrate reported by Calizo et al. [49] as well as graphene on a $\mathrm{SiO}_{2} / \mathrm{Si}$ substrate reported by Casiraghi et al. [50] are also included in Table 1. The positions of the $\mathrm{G}$ band $\left(1581 \mathrm{~cm}^{-1} \pm 1 \mathrm{~cm}^{-1}\right)$ and FWHM $\left(15.5 \mathrm{~cm}^{-1} \pm 1 \mathrm{~cm}^{-1}\right)$ are similar for graphene on $\mathrm{SiO}_{2}(300 \mathrm{~nm}) / \mathrm{Si}$, quartz, PDMS, Si, glass and NiFe substrates. The small differences in the $G$ band position on these substrates are within the range of fluctuation (1580-1588 $\mathrm{cm}^{-1}$ ) caused by unintentional electron or hole doping effects reported by Casiraghi et al. [50] for more than 40 graphene samples on a $\mathrm{SiO}_{2} / \mathrm{Si}$ substrate. Therefore, our observations indicate that the interaction between micromechanically cleaved graphene and different substrates is not strong enough to affect the physical structure of graphene. These results are consistent with those of Calizo et al. $[49,51]$ who suggested that the weak substrate effect can be explained by the fact that the $G$ band is made up of the long wavelength optical phonons (TO and LO) [41], and the out-of-plane vibrations in graphene are not coupled to this in-plane vibration [52]. On the other hand, for graphene grown on $\mathrm{SiC}$ substrates, it can be seen the intensity ratio of the $G$ and 2D bands of EMG differs significantly from those of SLG prepared by MC. Moreover, significant blueshifts of the $\mathrm{G}$ band $\left(11 \mathrm{~cm}^{-1}\right)$ and the $2 \mathrm{D}$ band $\left(\sim 34 \mathrm{~cm}^{-1}\right)$ of EMG are observed compared to those of graphene prepared by MC. This significant blueshift of the Raman bands can be understood in

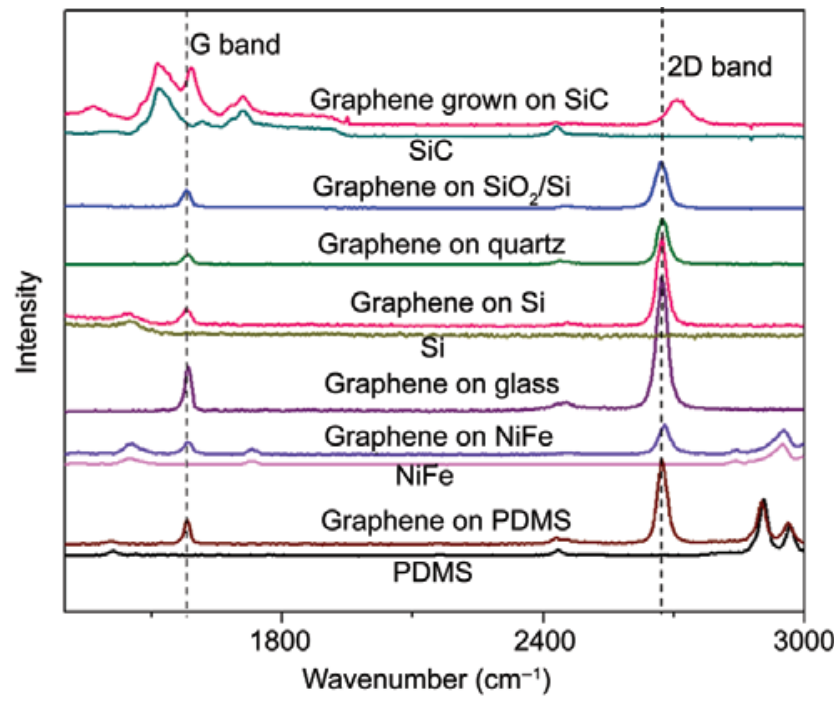

Figure 6 Raman spectra of SLG on different substrates as well that of epitaxial monolayer graphene on SiC. Reproduced with permission from J. Phys. Chem. C 2008, 112, 10637-10640. Copyright 2008 American Chemical Society [43]

Table 1 The G band and 2D band positions and their full width at half maximum (FWHM) for graphene/graphite on different substrates. Reproduced with permission from J. Phys. Chem. C 2008, 112, 10637-10640. Copyright 2008 American Chemical Society [43]

\begin{tabular}{ccccc}
\hline Substrate & $\begin{array}{c}\text { G band } \\
\text { position }\left(\mathrm{cm}^{-1}\right)\end{array}$ & G band FWHM $\left(\mathrm{cm}^{-1}\right)$ & 2D band position $\left(\mathrm{cm}^{-1}\right)$ & 2D band FWHM $\left(\mathrm{cm}^{-1}\right)$ \\
\hline $\mathrm{SiC}$ & 1591.5 & 31.3 & 2710.5 & 59.0 \\
$\mathrm{SiO}_{2} / \mathrm{Si}$ & 1580.8 & 14.2 & 2676.2 & 31.8 \\
$\mathrm{SiO}_{2} / \mathrm{Si}[50]$ & $1580-1588$ & $6-16$ & & 29.0 \\
Quartz & 1581.9 & 15.6 & 2674.6 & 28.3 \\
$\mathrm{Si}$ & 1580 & 16 & 2672 & 27 \\
PDMS & 1581.6 & 15.6 & 2673.6 & 30.8 \\
Glass & 1582.5 & 16.8 & 2672.8 & 31.4 \\
NiFe & 1582.5 & 14.9 & 2678.6 & 2.6 \\
GaAs [49] & 1580 & 15 & $2 \mathrm{D}_{1}: 2675.4$ & 27.4 \\
Graphite & 1580.8 & 16.0 & $2 \mathrm{D}_{2}: 272.8$ & 35.6 \\
\hline
\end{tabular}


terms of the strain effect generated by the substrate. Between EMG and the SiC substrate, there is an interfacial carbon layer, which has a graphene-like honeycomb lattice and is covalently bonded to the $\mathrm{SiC}$ substrate $[53,54]$. This will change the lattice constant as well as the electronic properties of the substrate. Therefore, the lattice mismatch between the graphene lattice and interfacial carbon layer may cause a compressive stress on EMG, giving rise to the shift in the Raman G band peak frequencies. Detailed studies of epitaxial graphene on a $\mathrm{SiC}$ substrate will be described in Section 7 [55].

On the basis of the Raman studies of SLG produced by $\mathrm{MC}$ on different substrates, we can conclude that the weak interactions between graphene sheets and the substrates play a negligible role in affecting the Raman features of the graphene sheets. Only EMG grown on a $\mathrm{SiC}$ substrate shows a strong blueshift of the $G$ band, which can be understood in terms of the strain effect caused by the strong interaction between graphene and the $\mathrm{SiC}$ substrate.

\section{Process-induced defects and strain on graphene}

In addition to global back gates, top local gates have been proposed for more complex graphene devices, such as p-n junctions [56], Veselago lenses [57], and Klein tunneling [58]. Oxides that have been used as top gates so far include $\mathrm{HfO}_{2}, \mathrm{Al}_{2} \mathrm{O}_{3}$, and $\mathrm{SiO}_{2}$. Although efforts have been made to deposit the gate insulator oxides without damaging the graphene or changing its electrical properties [56-58], the gate oxides will influence the graphene sheets in at least three ways: doping, defects, and various mechanical deformations. We present here a systematic study of graphene sheets subjected to defects and mechanical deformations induced by insulating capping layers using Raman spectroscopy and imaging [59].

Figure 7(a) shows the optical image of a graphene sample on the $\mathrm{SiO}_{2} / \mathrm{Si}$ substrate. The graphene sheet contains one, two, three, and four layers as determined by Raman and contrast methods [44].
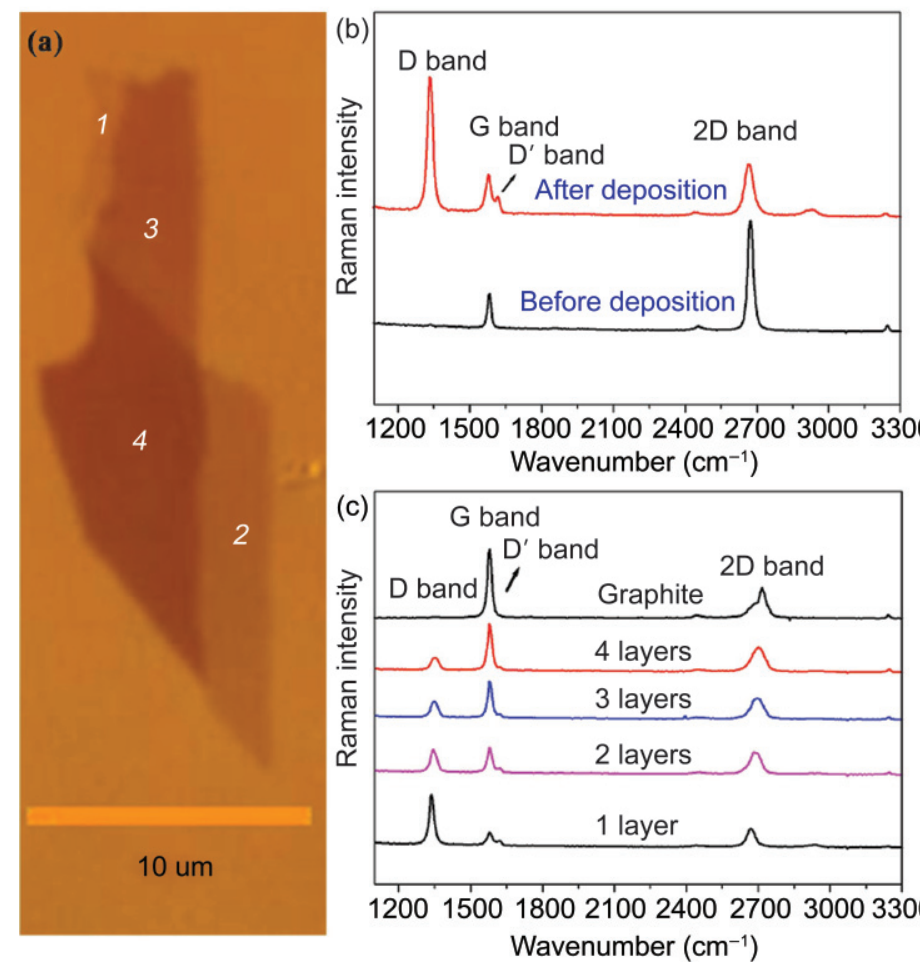
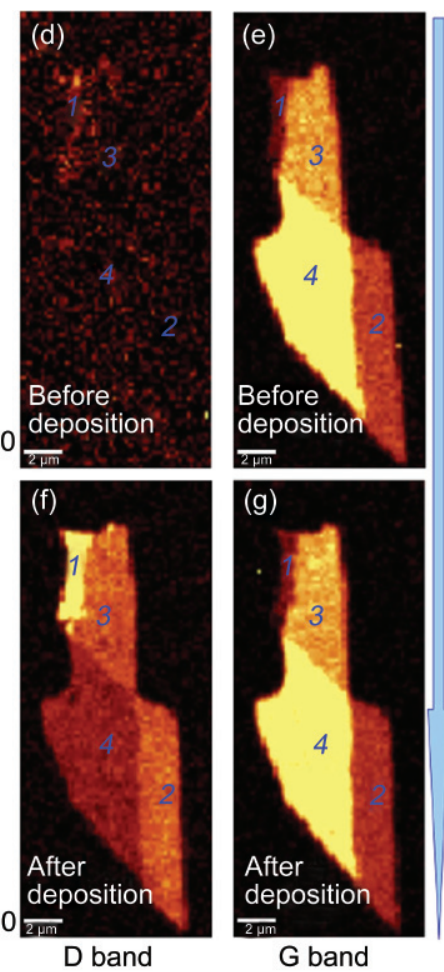

Figure 7 (a) Optical image of graphene sheets on a $\mathrm{SiO}_{2} / \mathrm{Si}$ substrate. (b) Raman spectra of SLG before and after deposition of the $5 \mathrm{~nm} \mathrm{SiO}$. (c) Raman spectra of graphene with different thicknesses as well as that of bulk graphite after $\mathrm{SiO}_{2}$ deposition. Raman images of graphene sheets before $\mathrm{SiO}_{2}$ deposition generated from the intensity of the $D$ band (d) and $G$ band (e). Raman images of graphene sheets after deposition of $5 \mathrm{~nm} \mathrm{SiO}_{2}$ using the intensity of D band (f), and G band (g). Reproduced with permission from ACS Nano 2008, 2, 1033 1039. Copyright 2008 American Chemical Society [59] 
A 5-nm $\mathrm{SiO}_{2}$ top layer was then deposited on the graphene by pulse laser deposition (PLD) with a 248-nm KrF pulsed laser. The laser power used was very weak $(\sim 200 \mathrm{~mJ}$ and repetition rate of 10 $\mathrm{Hz})$ to ensure a slow and smooth deposition (1 $\AA /$ min). The Raman spectra of graphene before and after $\mathrm{SiO}_{2}$ deposition are shown in Fig. 7(b). A clear difference is that two defect-induced Raman bands, denoted D $\left(1350 \mathrm{~cm}^{-1}\right)$ and D' $\left(1620 \mathrm{~cm}^{-1}\right)$ bands $[42,60]$, were observed after deposition. The observation of D and D' bands indicates that defects were introduced into the graphene after deposition of the $5 \mathrm{~nm} \mathrm{SiO}$ top layer. This may be caused by damage to the sample during deposition, or by the interaction between $\mathrm{SiO}_{2}$ and graphene which may produce vacancies, dislocation and/or dangling bonds. Annealing was carried out to eliminate the defects, as discussed below. Figure 7(c) shows the Raman spectra of a graphene sheet with one to four layers as well as that of bulk graphite after $\mathrm{SiO}_{2}$ deposition. The Raman spectra were recorded under same conditions. The D band intensity decreases with increasing graphene thickness and is invisible for bulk graphite, demonstrating that defects are more easily introduced into thinner graphene sheets [37]. Figures 7(d) and 7(f) show Raman images generated from the intensity of $\mathrm{D}$ band before and after deposition, respectively. Before deposition, there is no D band hence the Raman image is dark. After deposition, the thinner graphene (single layer graphene) shows the stronger D band, which is consistent with the discussion above. Figures 7(e) and $7(\mathrm{~g})$ show the images generated from the intensity of the corresponding $\mathrm{G}$ band, and they do not show any noticeable difference.

We have also deposited different materials as capping layers $\left(\mathrm{SiO}_{2}, \mathrm{HfO}_{2}, \mathrm{Si}\right.$, and polymethyl methacrylate (PMMA)) using different methods, such as electron beam evaporation, PLD, RF sputtering, and spin coating. Our results show that the deposition methods have a significant effect on the number of defects, with spin coating introducing the least amount of defects (no D band), followed by electron beam evaporation (weak D band). PLD and RF sputtering introduce the most defects (strong D band).

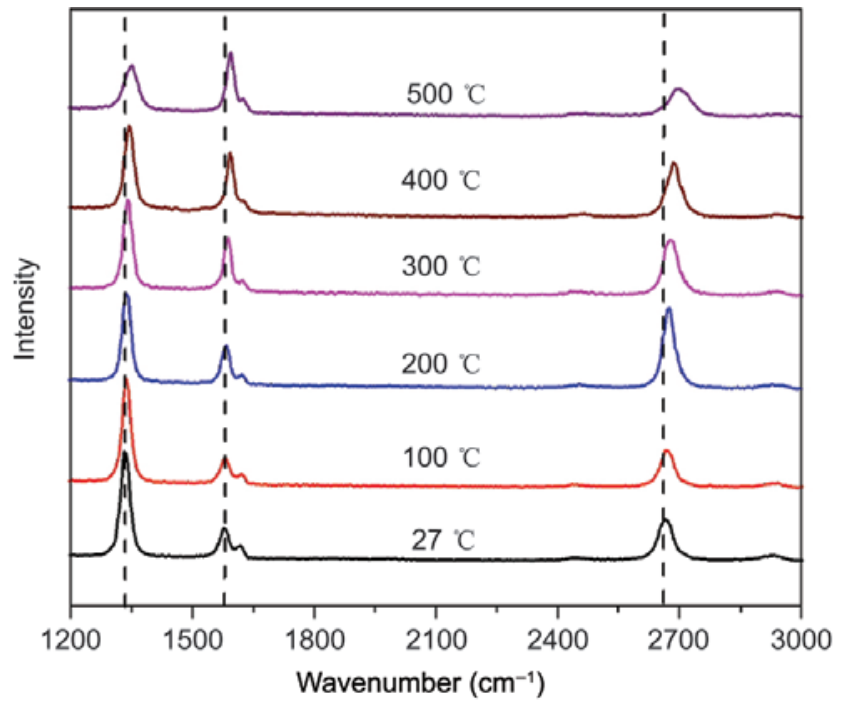

(a)

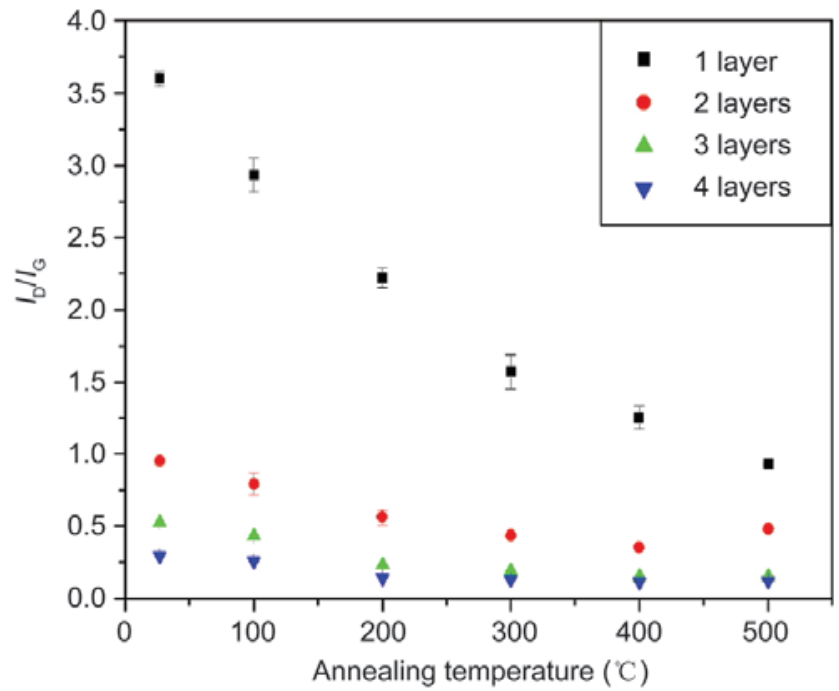

(b)

Figure 8 (a) Raman spectra of single layer graphene coated by $5 \mathrm{~nm}$ $\mathrm{SiO}_{2}$ and annealed at different temperatures. (b) The intensity ratio of the $D$ and $G$ bands $\left(I_{D} / I_{G}\right)$ of graphene sheets with one to four layers (coated with $\mathrm{SiO}_{2}$ ) after annealing at different temperatures. The ratio $I_{D} / I_{G}$ (corresponding to the number of defects) decreased significantly upon annealing. Reproduced with permission from ACS Nano 2008, 2, 1033-1039. Copyright 2008 American Chemical Society [59]

The Raman spectra of single layer graphene after $\mathrm{SiO}_{2}$ deposition and annealing in air at different temperatures are shown in Fig. 8(a). We also carried out vacuum annealing and similar results were observed. The intensity of the $\mathrm{D}$ band decreases after annealing. This is clearly demonstrated in Fig. 8(b), which shows the intensity ratio of the D and G bands $\left(I_{\mathrm{D}} / I_{\mathrm{G}}\right)$; this is often used to estimate the amount of defects in carbon materials. For one to four-layer 
graphene sheets, this ratio decreases with increasing annealing temperature. This can be understood as indicating the recovery of damaged graphene at high temperature. Another important observation in Fig. 8(a) is that all the Raman bands are shifted to higher frequency with increasing annealing temperature. Figure 9(a) shows the blueshift of the G band of the graphene sheets as the annealing temperature increases. The $\mathrm{G}$ band is blueshifted by $\sim 15 \mathrm{~cm}^{-1}$, whilst the D band is blueshifted by $\sim 13 \mathrm{~cm}^{-1}$ and the 2D band by $\sim 29 \mathrm{~cm}^{-1}$ after annealing at $500{ }^{\circ} \mathrm{C}$. We attribute this significant blueshift of the Raman bands to the strong compressive stress on graphene. The $\mathrm{SiO}_{2}$ becomes denser upon annealing so it exerts a strong compressive stress on the graphene. For comparison, the Raman bands of bulk graphite do not shift after deposition and annealing, which supports the above explanation, as bulk graphite is too thick and is not easily compressed by $\mathrm{SiO}_{2}$. Recently, Yan et al. [22] and Pisana et al. [23] found that the frequency of the G and 2D Raman bands can also be adjusted by charge doping through electron-phonon coupling changes. In addition to the blueshift of the $G$ band, a bandwidth narrowing of $\sim 10 \mathrm{~cm}^{-1}$ was also observed in the case of charge doping. However, in our results, only a small fluctuation $\left( \pm 1 \mathrm{~cm}^{-1}\right)$ of the FWHM (full width at half maximum) of the $G$ band was observed after annealing at different temperatures, which indicates that the effect of charge doping can be ignored. In addition, it has been shown that the dependence of the 2D band blueshift on doping is very weak and only $\sim 10 \%-30 \%$ of that of the $G$ band $[22,25]$. The blueshift of the $2 \mathrm{D}$ band of $25 \mathrm{~cm}^{-1}$ is therefore too large to result from charge doping alone. Therefore, it can be concluded that the observed shifts in the positions of the $\mathrm{G}\left(\sim 15 \mathrm{~cm}^{-1}\right)$ and $2 \mathrm{D}\left(\sim 25 \mathrm{~cm}^{-1}\right)$ bands in the above experiments were mainly caused by stress.

The compressive stress on graphene in the above experiments is due to the increase in the density of $\mathrm{SiO}_{2}$ upon annealing. The origin of the compressive stress is very similar to the biaxial stress resulting from the lattice mismatch at the sample/substrate interface in a normal thin film. Therefore, the stress on graphene should be biaxial. The biaxial compressive stress on graphene can be estimated from the shift of the Raman $E_{2 g}$ phonon. From a biaxial stress model, the $\mathrm{G}$ band stress coefficient of graphene is estimated [59] to be $7.47 \mathrm{~cm}^{-1} / \mathrm{GPa}$. The variation in stress on single layer graphene with annealing temperature can then be estimated and is shown in Fig. 9(b). The compressive stress on graphene was as high as $\sim 2.1$ GPa after depositing $\mathrm{SiO}_{2}$ and annealing at $500{ }^{\circ} \mathrm{C}$, and the stress on single layer graphene in our experiments can be fitted by the following formula:

$$
\sigma=-0.155+2.36 \times 10^{-3} \mathrm{~T}+5.17 \times 10^{-6} \mathrm{~T}^{2}
$$

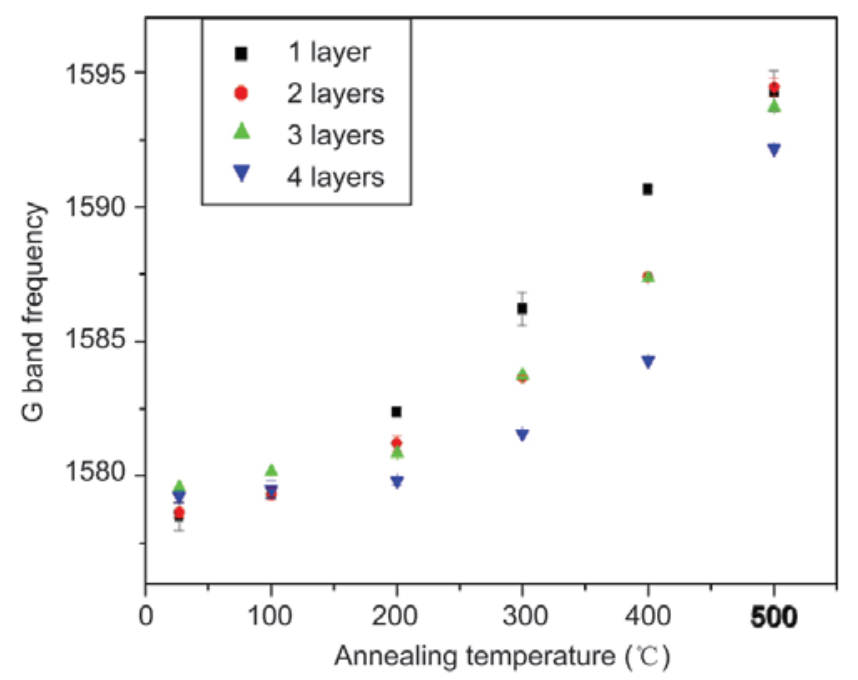

(a)

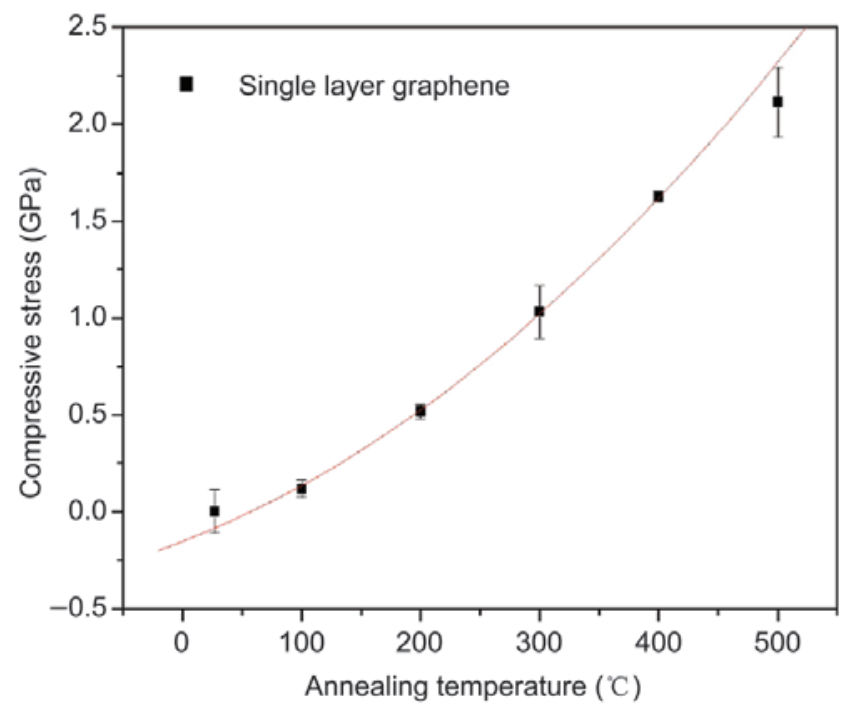

(b)

Figure 9 The Raman $\mathrm{G}$ band peak frequency of graphene sheets with one to four layers (coated with $\mathrm{SiO}_{2}$ ) after annealing at different temperatures. (b) Magnitude of compressive stress on single layer graphene controlled by annealing temperature. The red line is a curve fit to the experimental data. Reproduced with permission from ACS Nano 2008, 2, 1033-1039. Copyright 2008 American Chemical Society [59] 
where $\sigma$ is the compressive stress in GPa and $T$ is temperature in ${ }^{\circ} \mathrm{C}$. The appearance of such large stress is mainly because graphene sheets are very thin (0.325 nm in thickness for single layer graphene) [61], so that they can be easily compressed or expanded. We have also introduced tensile stress into graphene by depositing a thin cover layer of silicon. The G band of graphene was redshifted by $\sim 5 \mathrm{~cm}^{-1}$ after silicon deposition, which corresponds to a tensile stress of $\sim 0.67 \mathrm{GPa}$ on the graphene sheet. We suggest that tensile stress can be also achieved by depositing other materials with larger lattice constant than graphene. In combination with annealing, both compressive and tensile stress can be introduced into graphene and modified in a controllable manner. The stressed graphene may have very important applications as the properties of graphene (optical and electronic properties) can be adjusted by stress; stress studies in CNTs have already shown good examples of this [62 -64]. Stress engineering using SiGe alloys has already been used in IC fabrication in order to improve the device performance.

\section{Raman spectroscopy of folded graphene}

The peculiar properties of graphene are closely related to its unique electronic band structure. For SLG, it is known that the low energy dispersion is linear [65], which make charge carriers in SLG behave like massless Dirac-Fermions. Interestingly, recent studies such as magnetotransport [31], and far infrared magneto-transmission [66] investigations on multilayer epitaxial graphene (EG) with stacking disorder $[67,68]$ still revealed the two dimensional Dirac-like (SLG-like) character of the electronic states. Furthermore, theoretical calculations of the electronic structure of BLG with a twist in the second layer were carried out. The results showed that the low energy dispersion of twisted two-layer graphene is linear, as in SLG, but that the Fermi velocity is significantly smaller [69]. Further investigation of the electronic structure of BLG that deviates from $\mathrm{AB}$ stacking is necessary in order to obtain a fundamental understanding of the relationship between stacking order and electronic properties of bi- or multi- layer graphene.
We have successfully fabricated $1+1$ folded graphenes with different stacking orders and studied their electronic properties using Raman spectroscopy [70]. The 1+1 layer samples were prepared by simply gently flushing de-ionized water across the surface of the substrate containing the target graphene sheet. The 1+1 layer folded graphenes can be observed after this process. Figures 10(a) and (b) show the optical images of SLG before and after folding. The black dashed rectangle indicates the location where folding happens. As the optical images are not very clear, Figs. 10(c) and (d) show schematic drawings of the sample before and after folding. Figures 10(e) and (f) respectively show Raman images of $2 \mathrm{D}$ and $\mathrm{G}$ band positions (frequency) after folding. As shown in Fig. 10(e), the 2D band frequency of folded graphene is much higher than that of SLG. On the other hand, in Fig. 10(f), an even contrast can be seen which means there is almost no change in $\mathrm{G}$ band frequency before and after folding. Figure 10(g) shows the Raman image obtained from the 2D peak area (integrated intensity) after folding. The peak intensity for folded graphene is much higher than that for SLG. It has been observed in previous work [39] that the area of the 2D band is almost identical for 1 to 4 layer graphene. Hence, the much stronger 2D band of folded graphene reveals that its structure and properties are different from those of BLG. Figure 10(h) shows the Raman image extracted from the G band area after folding. The $G$ band intensity of the folded graphene is nearly double that of SLG since it contains two layers of carbon atoms.

Figure 11 gives typical Raman spectra of the $1+1$ folded graphene (taken from area $\mathbf{X}$ in Fig. 10(e)), SLG (taken from area Y in Fig. 10(e)) and BLG for comparison. The spectra are normalized to have similar $G$ band intensity. It can be seen that the Raman spectrum of $1+1$ folded graphene is different from that of BLG. The 2D band of BLG is much broader and can be fitted as four peaks, which originate from the splitting of the valence and conduction bands [19]. However, for $1+1$ folded graphene, only a single sharp peak is present which is similar to that of SLG. Thus, the electronic structure of folded graphene should be similar to that of SLG i.e., there is no splitting of energy bands. The similarity of the

\section{Springer}



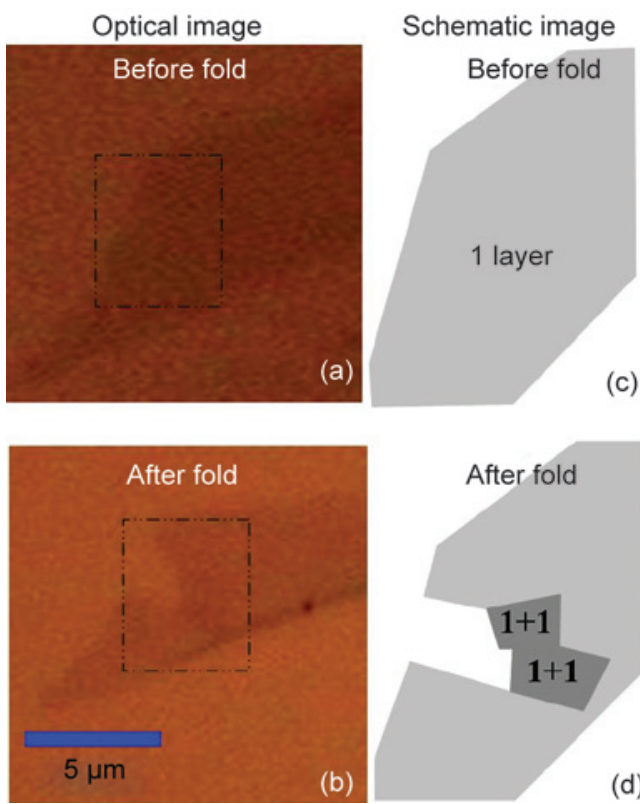

(c)

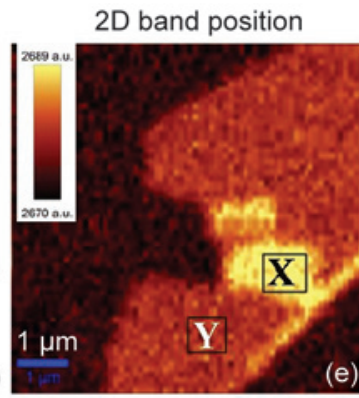

$\mathrm{G}$ band position

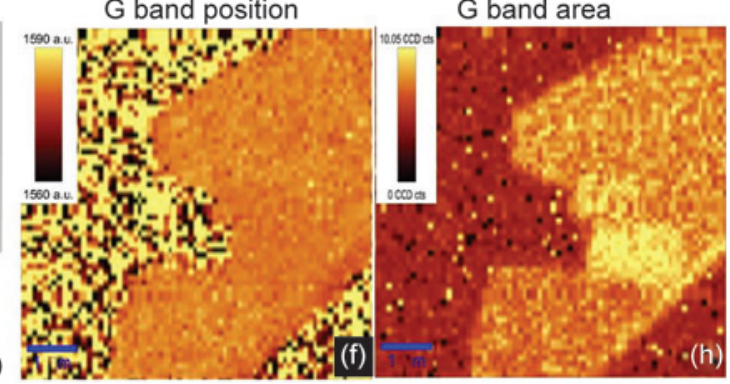

Figure 10 Optical images of SLG (a) before and (b) after folding. Schematic images of SLG (c) before and (d) after folding. Raman image obtaining from the (e) 2D and ( $f$ ) $G$ band positions. Raman images obtained by extracting the area of the (g) 2D and (h) G band. Reproduced with permission from Phys. Rev. B 2008, 77, 235403. Copyright 2008 American Physical Society [70]

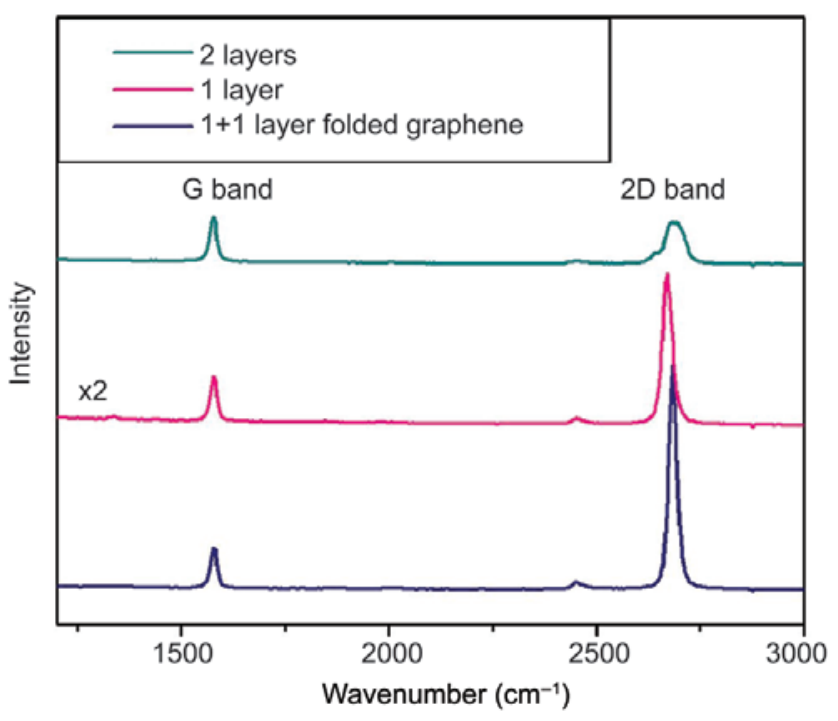

Figure 11 Raman spectra of BLG, SLG and 1+1 layer folded graphene. The spectra are normalized to have similar $G$ band intensity. Reproduced with permission from Phys. Rev. B 2008, 77, 235403. Copyright 2008 American Physical Society [70]

2D bands of folded graphene and SLG calls for care when using Raman to identify SLG. Although the Raman features of folded graphene are quite similar as those of SLG, there are differences that need to be noticed: a strong blueshift $\left(\sim 12 \mathrm{~cm}^{-1}\right)$ of the $2 \mathrm{D}$ band of folded graphene $\left(2686 \mathrm{~cm}^{-1}\right)$ compared to SLG (2674 $\mathrm{cm}^{-1}$ ) can be clearly seen, as indicated in the Raman images (Fig. 10(e)). This blueshift is associated with the SLG-like band structure of $1+1$ folded graphene but with smaller Fermi velocity, as will be discussed in detail later. The folded graphene has a higher 2D to G band intensity ratio than that of SLG, partially due to the different resonance conditions of folded graphene and SLG. We have studied a total of six 1+1 layer folded graphene samples. Blueshifts of the 2D band compared to SLG are observed for every sample, but the magnitude of the blueshift varies between 4 and $12 \mathrm{~cm}^{-1}$.

Lopes dos Santos et al. [69] calculated the electronic structure of twisted BLG and their results showed that it has an SLG-like linear dispersion with a reduced Fermi velocity. The $1+1$ folded graphene can be viewed as twisted BLG with an unknown twist angle. Therefore, the energy band structure of our folded graphene can be estimated using twisted BLG as a model. Figure 12 shows a schematic illustration of the electronic structure of $1+1$ folded graphene as well as SLG. The reduction in Fermi velocity $\left(v_{\mathrm{F}}{ }^{\prime}\right)$ of folded graphene corresponds to the smaller slope of the energy dispersion near the Dirac point. The second-order double resonance Stokes process for folded graphene and SLG are also 


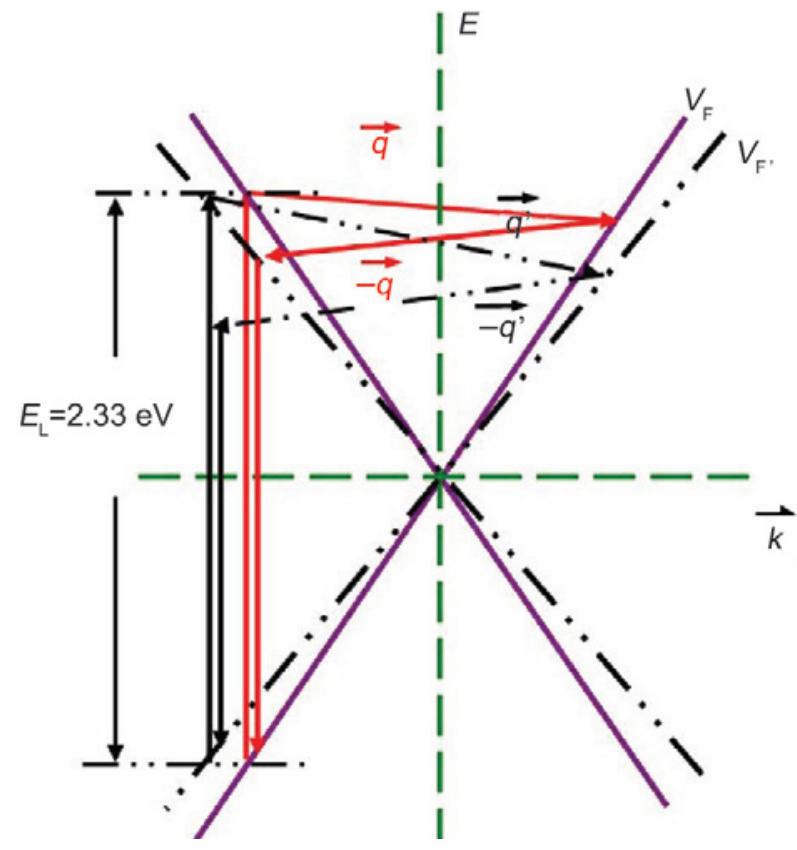

Figure 12 Schematic illustration of the electronic structure of 1+1 layer folded graphene (black dashed lines) as well as SLG (purple solid lines). The arrows indicate the double resonance process in folded graphene (black dashed lines) and SLG (red solid lines). Reproduced with permission from Phys. Rev. B 2008, 77, 235403. Copyright 2008 American Physical Society [70]

shown schematically in Fig. 12. For folded graphene, a phonon with larger wave vector $\left(\overrightarrow{q^{\prime}}\right)$ is needed to inelastically scatter the electron compared to that for SLG $(\vec{q})$. Because of the almost linear dispersion of the optical phonon branch around the $K$ point $\left(\frac{\mathrm{d} w}{\mathrm{~d} q}\right)$ [19] which contributes to the observed 2D band frequency, phonons with higher frequency $\left(\omega^{\prime}\right)$ are obtained in a double resonance process for folded graphene. This is the reason for the blueshift of the 2D band of folded graphene. By using this model, the sharpness and blueshift of the 2D band of multilayer EG on carbon-terminated SiC can be also easily understood [71], and attributed to the SLG-like linear dispersion of the energy bands of multi layer EG but with a $\sim 10 \%$ reduction in Fermi velocity $[66,72]$ relative to that of SLG.

Moreover, the Fermi velocity of folded graphene can be estimated. In the double resonance process [42], it is known that

$$
\hbar v_{\mathrm{F}} q \approx E_{\mathrm{L}}-\hbar(\omega / 2)
$$

where $E_{\mathrm{L}}$ is the incident photon energy, $v_{\mathrm{F}}$ is the Fermi velocity, $\omega$ is the frequency of the two phonon 2D band, and $\hbar(\omega / 2)$ is the phonon energy. With simple mathematics, the relation between the Fermi velocity change and $2 \mathrm{D}$ frequency shift can be derived [70]:

$$
\frac{\mathrm{d} v_{\mathrm{F}}}{v_{\mathrm{F}}}=-\frac{\hbar v_{\mathrm{F}}}{\left[E_{\mathrm{L}} v_{\mathrm{F}}-\hbar(\omega / 2)\right] \cdot \frac{\mathrm{d} \omega}{\mathrm{d} q}} \mathrm{~d} \omega
$$

where, $\hbar v_{\mathrm{F}}=6.5 \mathrm{eV} \cdot \AA$ [42], $E_{\mathrm{L}}=2.33 \mathrm{eV}, \hbar(\omega / 2)=0.166$ eV by taking $\omega \approx 2670 \mathrm{~cm}^{-1}$ and $\frac{\mathrm{d} \omega}{\mathrm{d} q}=645 \mathrm{~cm}^{-1} \cdot \AA$ is the phonon dispersion around the K point [19]. Therefore,

$$
\frac{\mathrm{d} v_{\mathrm{F}}}{v_{\mathrm{F}}}=-\frac{37.5 \cdot \mathrm{d} \omega}{\mathrm{eV}} \text { or }-\frac{0.00467 \cdot \mathrm{d} \omega}{\mathrm{cm}^{-1}}
$$

where $\frac{d v_{F}}{v_{F}}$ is the Fermi velocity change in percentage terms and $\mathrm{d} \omega$ is the frequency change of the 2D band (in $\mathrm{eV}$ or $\mathrm{cm}^{-1}$ ) after folding.

For the $1+1$ folded graphene samples, the maximum blueshift of the 2D band is $\sim 12 \mathrm{~cm}^{-1}$, and thus the reduction in Fermi velocity is about $5.6 \%$. The smallest blue shift $\left(\sim 4 \mathrm{~cm}^{-1}\right)$ corresponds to smaller reduction in Fermi velocity ( 2\%). The different values may be due to the different twist angles between the first and second graphene layers for different samples which is known [69] to affect the Fermi velocity and hence the blueshift of the 2D band. Another reason is the different separation of the two layers, which will also affect the electronic properties of folded graphene.

\section{Raman spectroscopy of epitaxial graphene}

Most of the Raman studies above were carried out on micromechanical cleavage graphene (MCG) [19, 37, 44]. In this section, we present Raman studies of epitaxial graphene (EG) grown on $\mathrm{SiC}$ substrates [55]. $\mathrm{EG}$ grown on $\mathrm{SiC}$ is suitable for large area fabrication and is more compatible with current Si processing techniques for future applications. Nevertheless, the EG may interact with the SiC substrate which could modify its optical and electronic properties. A bandgap of $\sim 0.26 \mathrm{eV}$ was observed by angleresolved photoemission spectroscopy (ARPES) for EG grown on a $\mathrm{SiC}$ substrate, which was attributed to the interaction of graphene with the substrate [73]. Some theoretical $[54,74]$ and experimental studies on EG e.g., X-ray diffraction (XRD) [54, 75] and scanning 
tunneling microscopy (STM) [76, 77], have also been carried out. However, the effect of $\mathrm{SiC}$ substrates on EG is still not well understood. The formation of graphene on a $\mathrm{SiC}$ substrate can be described as follows: the $\mathrm{SiC}$ surface first reconstructs to a $(\sqrt{3} \times$ $\sqrt{3}) \mathrm{R} 30^{\circ}$ (R3) structure, then to a $(6 \sqrt{3} \times 6 \sqrt{3}) \mathrm{R} 30^{\circ}(6 \mathrm{R} 3)$ structure, referred to here as a carbon nanomesh, and finally after annealing at higher temperature graphene forms on the carbon nanomesh. However, there is still some debate as to how the graphene bonds/connects to the $6 \mathrm{R} 3 /$ carbon nanomesh structure.

The EG samples were prepared by the following process: annealing a chemically etched (10\% HF solution) n-type Si-terminated $6 \mathrm{H}-\mathrm{SiC}$ (0001) sample (CREE Research Inc.) at $850{ }^{\circ} \mathrm{C}$ under a silicon flux for $2 \mathrm{~min}$ in ultrahigh vacuum (UHV) resulting in a Si-rich 3×3-reconstructed surface, and subsequently annealing the sample several times at $1300{ }^{\circ} \mathrm{C}$ in the absence of the silicon flux to form EG [31, 78]. The structure of EG was confirmed by in situ low energy electron diffraction (LEED), STM, and photoemission spectroscopy (PES) [76]. Since the characteristic STM images of carbon nanomesh and single layer graphene are quite different, the appearance of single layer EG can be detected by monitoring the phase evolution from carbon nanomesh to graphene by in situ STM during the thermal annealing of SiC $[76,79]$. However, the STM images for single layer and bilayer $\mathrm{EG}$ on $\mathrm{SiC}$ are very similar. It is very hard to determine the layer thickness using this method. Instead, layer thickness for bilayer or thicker graphene sample has been measured by monitoring the attenuation of the bulk SiC related Si $2 p$ PES signal (with a photon beam energy of $500 \mathrm{eV}$ ) under normal emission conditions [76].

Figure 13 shows the Raman spectra of single and two layer EG (grown on Si terminated SiC), MCG, bulk graphite, and the $\mathrm{SiC}$ substrate [55]. The $6 \mathrm{H}-\mathrm{SiC}$ has several overtone peaks in the range 1000 to 2000 $\mathrm{cm}^{-1}$. The peak near $1520 \mathrm{~cm}^{-1}$ is the overtone of the $\mathrm{TO}(\mathrm{X})$ phonon at $761 \mathrm{~cm}^{-1}$. The peak near $1713 \mathrm{~cm}^{-1}$ is a combination of optical phonons with wave vectors near the $M$ point at the zone edges [80,81]. The weak $\mathrm{SiC}$ peak at $\sim 1620 \mathrm{~cm}^{-1}$ is not observable in our EG samples. The Raman spectrum of single layer EG has five peaks, located at 1368, 1520, 1597, 1713, and 2715 $\mathrm{cm}^{-1}$, of which the peaks at 1520 and $1713 \mathrm{~cm}^{-1}$ are from the $\mathrm{SiC}$ substrate. The $1368 \mathrm{~cm}^{-1}$ peak is the socalled defect-induced D band; the $1597 \mathrm{~cm}^{-1}$ peak is the in-plane vibrational $\mathrm{G}$ band; and the $2715 \mathrm{~cm}^{-1}$ peak is the two-phonon 2D band [60]. The Raman signal of single layer MCG is much stronger ( 10 times) than that of EG on the $\mathrm{SiC}$ substrate. This can be explained by the interference enhancement of the Raman signal of graphene on a $300 \mathrm{~nm} \mathrm{SiO} / \mathrm{Si}$ substrate [40]. The Raman spectrum of EG shows the defect-induced D band, indicating that it contains defects, which may result from surface dislocations, corrugation, the interaction of graphene with substrate, or vacancies. The $2 \mathrm{D}$ band of single layer EG is broader than that of MCG $\left(60 \mathrm{~cm}^{-1}\right.$ as compared with $\left.30 \mathrm{~cm}^{-1}\right)$ [19], which can be explained by the poorer crystallinity of EG. However, compared to two layer EG, the 2D band of single layer EG is much narrower $\left(60 \mathrm{~cm}^{-1}\right.$ compared to $95 \mathrm{~cm}^{-1}$ ) and has a lower frequency $(2715$ $\mathrm{cm}^{-1}$ compared to $2736 \mathrm{~cm}^{-1}$ ), which are characteristics of single layer graphene. The Raman results are in agreement with the STM and PES results and confirm that the EG on $\mathrm{SiC}$ in Fig. 13 is single and two layer. Another important observation is that the G (1597 $\left.\mathrm{cm}^{-1}\right)$ and 2D $\left(2715 \mathrm{~cm}^{-1}\right)$ bands of single layer EG shift significantly towards higher frequency from the

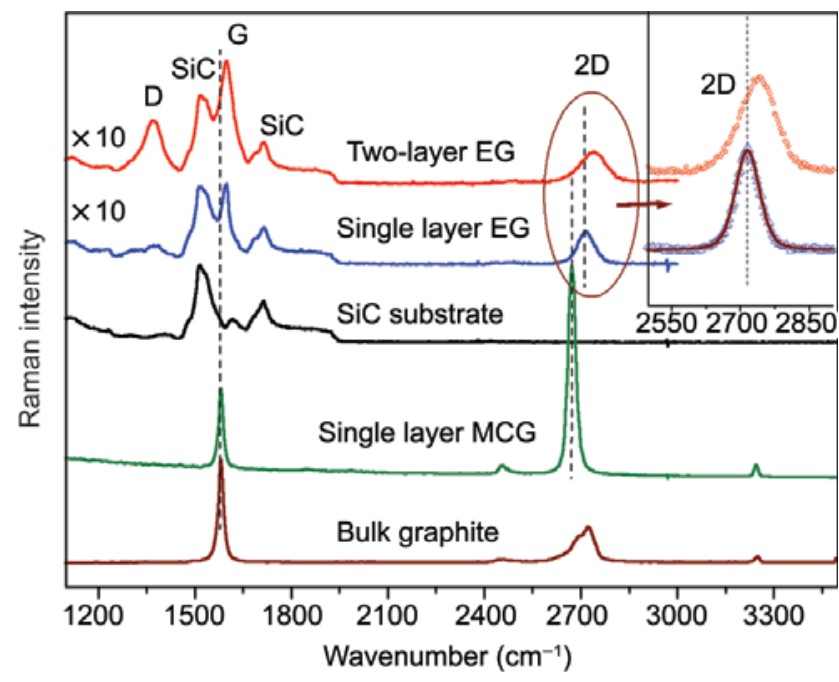

Figure 13 Raman spectra of single and two-layer EG grown on SiC, the $\mathrm{SiC}$ substrate, MCG, and bulk graphite as indicated. The inset is an enlarged part of the 2D band region of single and two layer EG. The hollow symbols are experimental data and the solid line is the fitted curve. Reproduced with permission from Phys. Rev. B 2008, 77, 115416. Copyright 2008 American Physical Society [55] 
positions of the $G\left(1580 \mathrm{~cm}^{-1}\right)$ and $2 \mathrm{D}\left(2673 \mathrm{~cm}^{-1}\right)$ bands of single layer MCG. Although the position of the G band of single and few layer MCG may fluctuate $\left( \pm 3 \mathrm{~cm}^{-1}\right)$ around the frequency of the $\mathrm{G}$ band of bulk graphite $\left(1580 \mathrm{~cm}^{-1}\right)$ while the $2 \mathrm{D}$ bands of MCG are located between 2660 and $2680 \mathrm{~cm}^{-1}$ [39], the significant shifts in the positions of the $G$ band (17 $\left.\mathrm{cm}^{-1}\right)$ and $2 \mathrm{D}$ band $\left(42 \mathrm{~cm}^{-1}\right)$ of EG are due to other mechanisms. The possibility that local electron/hole doping [22, 23, 25] in EG causes the Raman blueshift is not high, because the $2 \mathrm{D}$ band shift of $42 \mathrm{~cm}^{-1}$ is too large to be achieved by such doping. Instead, we attribute it to the interaction of the $\mathrm{SiC}$ substrate with EG, most probably the strain effect, whereby the strain changes the lattice constant of graphene, and hence the Raman peak frequencies [55].

To illustrate the origin of the strain, Fig. 14 shows a schematic image of a graphene layer on an $\mathrm{SiC}$ (0001) 6R3 reconstructed surface. The large black circles represent the $6 \mathrm{R} 3$ lattice. The bulk lattice constant we used for $\mathrm{SiC}$ was $3.073 \AA$ [82], while that for graphene was $2.456 \AA$ [83]. It is obvious that the $13 \times 13$ graphene (31.923 $\AA$ ) matches the 6R3 lattice (31.935 $\AA$ ) quite well [53]. On the other hand, the $2 \times$ 2 graphene $(4.9 \AA)$ does not match the R3 structure (5.34 Å) (small black circles). Previous STM results showed that the 6 R3 surface/carbon nanomesh

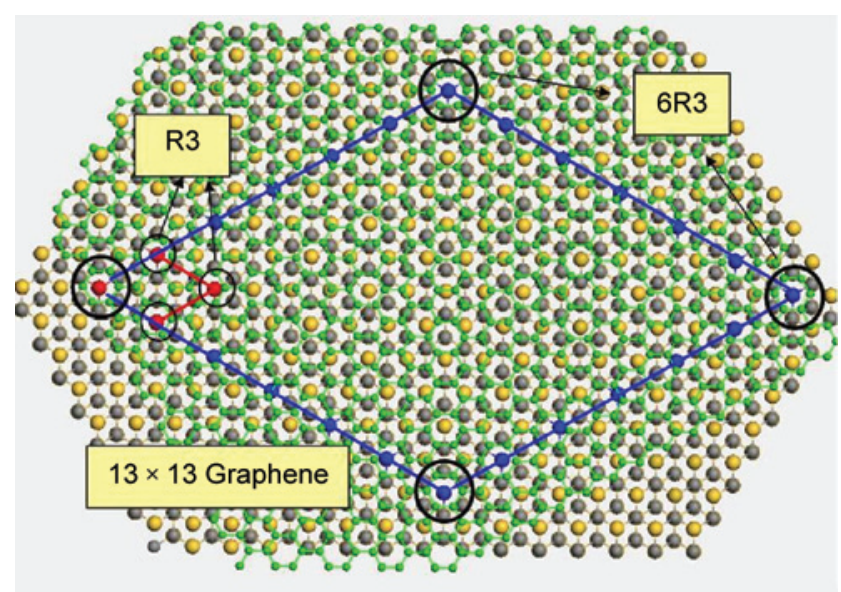

Figure 14 Schematic image of a graphene layer on a SiC (0001) surface. The green, yellow, and gray spheres represent $\mathrm{C}$ in graphene, $\mathrm{Si}$ in $\mathrm{SiC}$, and $\mathrm{C}$ in $\mathrm{SiC}$, respectively. The $\mathrm{SiC}$ surface was obtained after 6 R3 reconstruction and a $13 \times 13$ graphene lattice lies above it. The small black circles represent the R3 lattice, while the large black circles represent the $6 \mathrm{R} 3$ lattice. Reproduced with permission from Phys. Rev. B 2008, 77, 115416. Copyright 2008 American Physical Society [55] did not always retain its " $6 \times 6$ " periodicity. The pore size of the honeycombs observed by STM can be changed from $20 \AA$ to $30 \AA$, depending on the annealing temperature [76]. Hence, this surface can be described as a dynamic superstructure formed by the self-organization of surface carbon atoms at high temperatures. As a result, the mismatch between graphene $13 \times 13$ lattice $(\sim 32 \AA)$ and 6R3 surface/ carbon nanomesh (20 to $30 \AA$ ) will cause a compressive strain on EG. Recently, Rohrl et al. [84] also observed a blueshift of Raman bands of EG which they attributed to the compressive strain caused by the different thermal expansion coefficients of EG and $\mathrm{SiC}$. This might be another origin of compressive strain. However, attribution to the lattice mismatch (32 A compared to 20-30 ̊) between graphene and carbon nanomesh cannot be excluded [84].

Graphene has a very thin (2D structure) and the stress induced by the lattice mismatch with the $\mathrm{SiC}$ substrate can be considered as biaxial. The biaxial compressive stress on EG can also be estimated from the shift of Raman $E_{2 \mathrm{~g}}$ phonon ( $G$ band) with the stress coefficient about $7.47 \mathrm{~cm}^{-1} / \mathrm{GPa}$ [55]. Hence, the $17 \mathrm{~cm}^{-1}$ shift in the $\mathrm{G}$ band frequency of EG compared to that of bulk graphite or MCG corresponds to a biaxial stress of 2.27 GPa on EG. The strong compressive stress may affect both the physical and electronic properties of graphene, analogous to what occurs for CNTs [63, 64]. Raman spectra of few layer epitaxial graphene on $\mathrm{SiC}$ substrates have also been reported by Faugeras et al. [71] and no blueshift of the $\mathrm{G}$ band was observed. This may be because the epitaxial graphene they used was too thick (five to ten layers) and, as a consequence, the effect of the substrate on graphene is too weak to be observed.

\section{Conclusions}

A review of Raman spectroscopy and imaging of graphene has been presented. Raman and contrast spectroscopy can be used as an unambiguous method to determine the number of layers of graphene. The very strong Raman signal of single layer graphene, which is comparable to that of graphite, can be explained by an interference enhancement model. The effect of substrates on graphene has

\section{递量 Springer}


also been investigated. The results suggest that the weak interactions between graphene sheets and the substrates play a negligible role in affecting the Raman features of graphene sheets made by micromechanical cleavage. We also present the first experimental study of process-induced defects and stress in graphene using Raman spectroscopy and imaging. While defects lead to the observation of defect-related Raman bands, stress causes a shift in phonon frequency. A compressive stress (as high as $2.1 \mathrm{GPa}$ ) is induced in graphene by depositing a $5 \mathrm{~nm} \mathrm{SiO}$ coating followed by annealing. Raman spectroscopy has also been used to investigate the electronic structure of $1+1$ folded graphene that deviates from $\mathrm{AB}$ stacking. The $1+1$ folded graphene has a $2 \mathrm{D}$ band (sharp and symmetric) similar to that of SLG but has a blueshift (4 to $12 \mathrm{~cm}^{-1}$ ), which is due to the SLG-like electronic structure but with reduction in Fermi velocity. The similarity of the 2D bands of folded graphene and SLG calls for care when using Raman spectroscopy to identify SLG. Finally, Raman spectroscopy of epitaxial graphene grown on a $\mathrm{SiC}$ substrate was carried out. All the Raman peaks of epitaxial graphene have been assigned and compared with those of micromechanical cleaved graphene and bulk graphite. The results show that graphene grown on $\mathrm{SiC}$ is compressive stressed.

\section{References}

[1] Geim, A. K.; Novoselov, K. S. The rise of graphene. Nat. Mater. 2007, 6, 183-191.

[2] Novoselov, K. S.; Geim, A. K.; Morozov, S. V.; Jiang, D.; Zhang, Y.; Dubonos, S. V.; Grigorieva, I. V.; Firsov, A. A. Electric field effect in atomically thin carbon films. Science 2004, 306, 666-669.

[3] Zhang, Y.; Tan, Y. W.; Stormer, H. L.; Kim, P. Experimental observation of the quantum Hall effect and Berry's phase in graphene. Nature 2005, 438, 201-204.

[4] Novoselov, K. S.; Jiang, Z.; Zhang, Y.; Morozov, S. V.; Stormer, H. L.; Zeitler, U.; Maan, J. C.; Boebinger, G. S.; Kim, P.; Geim, A. K. Room-temperature quantum Hall effect in graphene. Science 2007, 315, 1379-1379.

[5] Bunch, J. S.; van der Zande, A. M.; Verbridge, S. S.; Frank, I. W.; Tanenbaum, D. M.; Parpia, J. M.; Craighead, H. G.; McEuen, P. L. Electromechanical resonators from graphene sheets. Science 2007, 315, 490-493.

[6] Heersche, H. B.; Jarillo-Herrero, P.; Oostinga, J. B.; Vandersypen, L. M. K.; Morpurgo, A. F. Bipolar supercurrent in graphene. Nature 2007, 446, 56-59.

[7] Ohta, T.; Bostwick, A.; Seyller, T.; Horn, K.; Rotenberg, E. Controlling the electronic structure of bilayer graphene. Science 2006, 313, 951-954.

[8] Meyer, J. C.; Geim, A. K.; Katsnelson, M. I.; Novoselov, K. S.; Booth, T. J.; Roth, S. The structure of suspended graphene sheets. Nature 2007, 446, 60-63.

[9] Bolotin, K. I.; Sikes, K. J.; Hone, J.; Stormer, H. L.; Kim, P. Temperature dependent transport in suspended graphene. Phy. Rev. Lett. 2008, 101, 096802.

[10] Bolotin, K. I.; Sikes, K. J.; Jiang, Z.; Fundenberg, G.; Hone, J.; Kim, P.; Stormer, H. L. Ultrahigh electron mobility in suspended graphene. Solid State Commun. 2008, 146, 351-355.

[11] Son, Y. W.; Cohen, M. L.; Louie, S. G. Half-metallic graphene nanoribbons. Nature 2007, 446, 342-342.

[12] Trauzettel, B.; Bulaev, D. V.; Loss, D.; Burkard, G. Spin qubits in graphene quantum dots. Nat. Phys. 2007, 3, 192-196.

[13] Blake, P.; Brimicombe, P. D.; Nair, R. R.; Booth, T. J.; Jiang, D.; Schedin, F.; Ponomarenko, L. A.; Morozov, S. V.; Gleeson, H. F.; Hill, E. W.; Geim, A. K.; Novoselov, K. S. Graphene-based liquid crystal device. Nano Lett. 2008, 8, 1704-1708.

[14] Nair, R. R.; Blake, P.; Grigorenko, A. N.; Novoselov, K. S.; Booth, T. J.; Stauber, T.; Peres, N. M. R.; Geim, A. K. Fine structure constant defines visual transparency of graphene. Science 2008, 320, 1308-1308.

[15] Stankovich, S.; Dikin, D. A.; Dommett, G. H. B.; Kohlhaas, K. M.; Zimney, E. J.; Stach, E. A.; Piner, R. D.; Nguyen, S. T.; Ruoff, R. S. Graphene-based composite materials. Nature 2006, 442, 282-286.

[16] Reich, S.; Thomsen, C. Raman spectroscopy of graphite. Philos. T. R. Soc. A 2004, 362, 2271-2288.

[17] Dresselhaus, M. S.; Eklund, P. C. Phonons in carbon nanotubes. Adv. Phys. 2000, 49, 705-814.

[18] Dresselhaus, M. S.; Dresselhaus, G.; Saito, R.; Jorio, A. Raman spectroscopy of carbon nanotubes. Phys. Rep. 2005, 409, 47-99.

[19] Ferrari, A. C.; Meyer, J. C.; Scardaci, V.; Casiraghi, C.; Lazzeri, M.; Mauri, F.; Piscanec, S.; Jiang, D.; Novoselov, K. S.; Roth, S.; Geim, A. K. Raman spectrum of graphene and graphene layers. Phys. Rev. Lett. 2006, 97, 187401. 
[20] Cancado, L. G.; Reina, A.; Kong, J.; Dresselhaus, M. S. Geometrical approach for the study of $G^{\prime}$ band in the Raman spectrum of monolayer graphene, bilayer graphene, and bulk graphite. Phys. Rev. B 2008, 77, 245408.

[21] Malard, L. M.; Nilsson, J.; Elias, D. C.; Brant, J. C.; Plentz, F.; Alves, E. S.; Castro, A. H.; Pimenta, M. A. Probing the electronic structure of bilayer graphene by Raman scattering. Phys. Rev. B 2007, 76, 201401.

[22] Yan, J.; Zhang, Y. B.; Kim, P.; Pinczuk, A. Electric field effect tuning of electron-phonon coupling in graphene. Phys. Rev. Lett. 2007, 98, 166802.

[23] Pisana, S.; Lazzeri, M.; Casiraghi, C.; Novoselov, K. S.; Geim, A. K.; Ferrari, A. C.; Mauri, F. Breakdown of the adiabatic Born-Oppenheimer approximation in graphene. Nat. Mater. 2007, 6, 198-201.

[24] Ferrari, A. C. Raman spectroscopy of graphene and graphite: Disorder, electron-phonon coupling, doping and nonadiabatic effects. Solid State Commun. 2007, 143, 47-57.

[25] Das, A.; Pisana, S.; Chakraborty, B.; Piscanec, S.; Saha, S. K.; Waghmare, U. V.; Novoselov, K. S.; Krishnamurthy, H. R.; Geim, A. K.; Ferrari, A. C.; Sood, A. K. Monitoring dopants by Raman scattering in an electrochemically top-gated graphene transistor. Nat. Nanotechnol. 2008, 3, 210-215.

[26] Stampfer, C.; Molitor, F.; Graf, D.; Ensslin, K.; Jungen, A.; Hierold, C.; Wirtz, L. Raman imaging of doping domains in graphene on $\mathrm{SiO}_{2}$. Appl. Phys. Lett. 2007, 91, 241907.

[27] Liu, L.; Ryu, S. M.; Tomasik, M. R.; Stolyarova, E.; Jung, N.; Hybertsen, M. S.; Steigerwald, M. L.; Brus, L. E.; Flynn, G. W. Graphene oxidation: Thickness-dependent etching and strong chemical doping. Nano Lett. 2008, 8, 19651970.

[28] Calizo, I.; Balandin, A. A.; Bao, W.; Miao, F.; Lau, C. $\mathrm{N}$. Temperature dependence of the Raman spectra of graphene and graphene multilayers. Nano Lett. 2007, 7, 2645-2649.

[29] Balandin, A. A.; Ghosh, S.; Bao, W. Z.; Calizo, I.; Teweldebrhan, D.; Miao, F.; Lau, C. N. Superior thermal conductivity of single-layer graphene. Nano Lett. 2008, 8, 902-907.

[30] Berger, C.; Song, Z. M.; Li, T. B.; Li, X. B.; Ogbazghi, A. Y.; Feng, R.; Dai, Z. T.; Marchenkov, A. N.; Conrad, E. H.; First, P.N.; de Heer, W. A. Ultrathin epitaxial graphite: 2D electron gas properties and a route toward graphene- based nanoelectronics. J. Phys. Chem. B 2004, 108, 19912-19916.

[31] Berger, C.; Song, Z. M.; Li, X. B.; Wu, X. S.; Brown, N.; Naud, C.; Mayo, D.; Li, T. B.; Hass, J.; Marchenkov, A. N.; Conrad, E. H.; First, P. N.; de Heer, W. A. Electronic confinement and coherence in patterned epitaxial graphene. Science 2006, 312, 1191-1196.

[32] Sutter, P. W.; Flege, J. I.; Sutter, E. A. Epitaxial graphene on ruthenium. Nat. Mater. 2008, 7, 406-411.

[33] Dedkov, Y. S.; Fonin, M.; Rudiger, U.; Laubschat, C. Rashba effect in the graphene/Ni(111) system. Phys. Rev. Lett. 2008, 100, 107602.

[34] Hernandez, Y.; Nicolosi, V.; Lotya, M.; Blighe, F.; Sun, Z.; De, S.; McGovern, I. T.; Holland, B.; Byrne, M.; Gun'Ko, Y.K.; Boland, J. J.; Niraj, P.; Duesberg, G.; Krishnamurti, S.; Goodhue, R.; Hutchison, J.; Scardaci, V.; Ferrari, A. C.; Coleman, J. N. High yield production of graphene by liquid phase exfoliation of graphite Nat. Nanotechnol. 2008, 3, 563-568.

[35] Li, X. L.; Wang, X. R.; Zhang, L.; Lee, S.W.; Dai, H. J. Chemically derived, ultrasmooth graphene nanoribbon semiconductors. Science 2008, 319, 1229-1232.

[36] Li, D.; Muller, M. B.; Gilje, S.; Kaner, R. B.; Wallace, G. G. Processable aqueous dispersions of graphene nanosheets. Nat. Nanotechnol. 2008, 3, 101-105.

[37] Gupta, A.; Chen, G.; Joshi, P.; Tadigadapa, S.; Eklund, P. C. Raman scattering from high-frequency phonons in supported n-graphene layer films. Nano Lett. 2006, 6, 2667-2673.

[38] Novoselov, K. S.; McCann, E.; Morozov, S. V.; Fal'ko, V. I.; Katsnelson, M. I.; Zeitler, U.; Jiang, D.; Schedin, F.; Geim, A. $K$ K. Unconventional quantum Hall effect and Berry's phase of $2 \pi$ in bilayer graphene. Nat. Phys. 2006, 2, 177-180.

[39] Graf, D.; Molitor, F.; Ensslin, K.; Stampfer, C.; Jungen, A.; Hierold, C.; Wirtz, L. Spatially resolved Raman spectroscopy of single- and few-layer graphene. Nano Lett. 2007, 7, 238-242.

[40] Wang, Y. Y.; Ni, Z. H.; Shen, Z. X.; Wang, H. M.; Wu, Y. H. Interference enhancement of Raman signal of graphene. Appl. Phys. Lett. 2008, 92, 043121.

[41] Pimenta, M. A.; Dresselhaus, G.; Dresselhaus, M. S.; Cancado, L. G.; Jorio, A.; Saito, R. Studying disorder in graphite-based systems by Raman spectroscopy. Phys. Chem. Chem. Phys. 2007, 9, 1276-1291.

[42] Thomsen, C.; Reich, S. Double resonant Raman scattering in graphite. Phys. Rev. Lett. 2000, 85, 5214-

\section{凰商: Springer}


5217.

[43] Wang, Y. Y.; Ni, Z. H.; Yu, T.; Wang, H. M.; Wu, Y. H.; Chen, W.; Wee, A. T. S.; Shen, Z. X. Raman studies of monolayer graphene: the substrate effect. J. Phys. Chem. C 2008, 112, 10637-10640.

[44] Ni, Z. H.; Wang, H. M.; Kasim, J.; Fan, H. M.; Yu, T.; Wu, Y. H.; Feng, Y. P.; Shen, Z. X. Graphene thickness determination using reflection and contrast spectroscopy. Nano Lett. 2007, 7, 2758-2763.

[45] Blake, P.; Hill, E. W.; Neto, A. H. C.; Novoselov, K. S.; Jiang, D.; Yang, R.; Booth, T. J.; Geim, A. K. Making graphene visible. Appl. Phys. Lett. 2007, 91, 063124.

[46] Roddaro, S.; Pingue, P.; Piazza, V.; Pellegrini, V.; Beltram, F. The optical visibility of graphene: Interference colors of ultrathin graphite on $\mathrm{SiO}_{2}$. Nano Lett. 2007, 7, 27072710.

[47] Casiraghi, C.; Hartschuh, A.; Lidorikis, E.; Qian, H.; Harutyunyan, H.; Gokus, T.; Novoselov, K. S.; Ferrari, A. $C$. Rayleigh imaging of graphene and graphene layers. Nano Lett. 2007, 7, 2711-2717.

[48] Palik, E. D. Handbook of Optical Constants of Solids; Academic Press: New York, 1991.

[49] Calizo, I.; Bao, W. Z.; Miao, F.; Lau, C. N.; Balandin, A. A. The effect of substrates on the Raman spectrum of graphene: Graphene-on-sapphire and graphene-onglass. Appl. Phys. Lett. 2007, 91, 201904.

[50] Casiraghi, C.; Pisana, S.; Novoselov, K. S.; Geim, A. K.; Ferrari, A. C. Raman fingerprint of charged impurities in graphene. Appl. Phys. Lett. 2007, 91, 233108.

[51] Calizo, I.; Teweldebrhan, D.; Bao, W.; Miao, F.; Lau, C. N.; Balandin, A. A. Spectroscopic Raman nanometrology of graphene and graphene multilayers on arbitrary substrates. J. Phys.: Conf. Ser. 2008, 109, 012008.

[52] Falkovsky, L. A. Phonon dispersion in graphene. J. Exp. Theor. Phys. 2007, 105, 397-403.

[53] Kim, S.; Ihm, J.; Choi, H. J.; Son, Y. W. Origin of anomalous electronic structures of epitaxial graphene on silicon carbide. Phys. Rev. Lett. 2008, 100, 176802.

[54] Varchon, F.; Feng, R.; Hass, J.; Li, X.; Nguyen, B. N.; Naud, C.; Mallet, P.; Veuillen, J. Y.; Berger, C.; Conrad, E. $\mathrm{H}$.; Magaud, L. Electronic structure of epitaxial graphene layers on SiC: Effect of the substrate. Phys. Rev. Lett. 2007, 99, 126805.

[55] Ni, Z. H.; Chen, W.; Fan, X. F.; Kuo, J. L.; Yu, T.; Wee, A. T. S.; Shen, Z. X. Raman spectroscopy of epitaxial graphene on a SiC substrate. Phys. Rev. B 2008, 77, 115416.
[56] Ozyilmaz, B.; Jarillo-Herrero, P.; Efetov, D.; Abanin, D. A.; Levitov, L. S.; Kim, P. Electronic transport and quantum Hall effect in bipolar graphene p-n-p junctions. Phys. Rev. Lett. 2007, 99, 166804.

[57] Cheianov, V. V.; Fal'ko, V.; Altshuler, B. L. The focusing of electron flow and a Veselago lens in graphene $p-n$ junctions. Science 2007, 315, 1252-1255.

[58] Katsnelson, M. I.; Novoselov, K. S.; Geim, A. K. Chiral tunnelling and the Klein paradox in graphene. Nat. Phys. 2006, 2, 620-625.

[59] Ni, Z. H.; Wang, H. M.; Ma, Y.; Kasim, J.; Wu, Y. H.; Shen, Z. X. Tunable stress and controlled thickness modification in graphene by annealing. ACS Nano 2008, 2, 1033-1039.

[60] Barros, E. B.; Demir, N. S.; Filho, A. G. S.; Filho, J. M.; Jorio, A.; Dresselhaus, G.; Dresselhaus, M. S. Raman spectroscopy of graphitic foams. Phys. Rev. B 2005, 71, 165422.

[61] Dresselhaus, M. S.; Dresselhaus, G.; Eklund, P. C. Science of Fullerenes and Carbon Nanotubes; Academic Press: San Diego, CA., 1996, p. 965.

[62] Maiti, A.; Svizhenko, A.; Anantram, M. P. Electronic transport through carbon nanotubes: Effects of structural deformation and tube chirality. Phys. Rev. Lett. 2002, 88, 126805.

[63] Minot, E. D.; Yaish, Y.; Sazonova, V.; Park, J. Y.; Brink, M.; McEuen, P. L. Tuning carbon nanotube band gaps with strain. Phys. Rev. Lett. 2003, 90, 156401.

[64] Heyd, R.; Charlier, A.; McRae, E. Uniaxial-stress effects on the electronic properties of carbon nanotubes. Phys. Rev. B 1997, 55, 6820-6824.

[65] Novoselov, K. S.; Geim, A. K.; Morozov, S. V.; Jiang, D.; Katsnelson, M. I.; Grigorieva, I. V.; Dubonos, S. V.; Firsov, A. A. Two-dimensional gas of massless Dirac fermions in graphene. Nature 2005, 438, 197-200.

[66] Sadowski, M. L.; Martinez, G.; Potemski, M.; Berger, C.; de Heer, W. A. Landau level spectroscopy of ultrathin graphite layers. Phys. Rev. Lett. 2006, 97, 266405.

[67] Hass, J.; Feng, R.; Li, T.; Li, X.; Zong, Z.; de Heer, W. A.; First, P. N.; Conrad, E. H.; Jeffrey, C. A.; Berger, C. Highly ordered graphene for two dimensional electronics. Appl. Phys. Lett. 2006, 89, 143106.

[68] Hass, J.; Varchon, F.; Millan-Otoya, J. E.; Sprinkle, M.; Sharma, N.; De Heer, W. A.; Berger, C.; First, P. N.; Magaud, L.; Conrad, E. H. Why multilayer graphene on $4 \mathrm{H}-\mathrm{SiC}(0001)$ behaves like a single sheet of graphene. 
Phys. Rev. Lett. 2008, 100, 125504.

[69] Lopes dos Santos, J. M. B.; Peres, N. M. R.; Castro, A. H. Graphene bilayer with a twist: Electronic structure. Phys. Rev. Lett. 2007, 99, 256802.

[70] Ni, Z. H.; Wang, Y. Y.; Yu, T.; You, Y. M.; Shen, Z. X. Reduction of Fermi velocity in folded graphene observed by resonance Raman spectroscopy. Phys. Rev. B 2008, 77, 235403.

[71] Faugeras, C.; Nerriere, A.; Potemski, M.; Mahmood, A.; Dujardin, E.; Berger, C.; de Heer, W. A. Few-layer graphene on $\mathrm{SiC}$, pyrolitic graphite, and graphene: A Raman scattering study. Appl. Phys. Lett. 2008, 92, 011914.

[72] Plochocka, P.; Faugeras, C.; Orlita, M.; Sadowski, M. L.; Martinez, G.; Potemski, M.; Goerbig, M. O.; Fuchs, J. N.; Berger, C.; de Heer, W. A. High-energy limit of massless Dirac Fermions in multilayer graphene using magnetooptical transmission spectroscopy. Phys. Rev. Lett. 2008, 100, 087401.

[73] Zhou, S. Y.; Gweon, G. H.; Fedorov, A. V.; First, P. N.; De Heer, W. A.; Lee, D. H.; Guinea, F.; Neto, A. H. C.; Lanzara, A. Substrate-induced bandgap opening in epitaxial graphene. Nat. Mater. 2007, 6, 770-775.

[74] Mattausch, A.; Pankratov, O. Ab initio study of graphene on SiC. Phys. Rev. Lett. 2007, 99, 076802.

[75] Hass, J.; Feng, R.; Millan-Otoya, J. E.; Li, X.; Sprinkle, M.; First, P. N.; de Heer, W. A.; Conrad, E. H.; Berger, C. Structural properties of the multilayer graphene/4H$\mathrm{SiC}(0001)$ system as determined by surface $\mathrm{X}$-ray diffraction. Phys. Rev. B 2007, 75, 214109.
[76] Chen, W.; Xu, H.; Liu, L.; Gao, X. Y.; Qi, D. C.; Peng, G. W.; Tan, S. C.; Feng, Y. P.; Loh, K. P.; Wee, A. T. S. Atomic structure of the $6 \mathrm{H}-\mathrm{SiC}(0001)$ nanomesh. Surf. Sci. 2005, 596, 176-186.

[77] Mallet, P.; Varchon, F.; Naud, C.; Magaud, L.; Berger, C.; Veuillen, J. Y. Electron states of mono- and bilayer graphene on SiC probed by scanning-tunneling microscopy. Phys. Rev. B 2007, 76, 041403.

[78] Chen, W.; Chen, S.; Qi, D. C.; Gao, X. Y.; Wee, A. T. S. Surface transfer p-type doping of epitaxial graphene. J. Am. Chem. Soc. 2007, 129, 10418-10422.

[79] Riedl, C.; Starke, U.; Bernhardt, J.; Franke, M.; Heinz, K. Structural properties of the graphene-SiC(0001) interface as a key for the preparation of homogeneous large-terrace graphene surfaces. Phys. Rev. B 2007, 76, 245406

[80] Burton, J. C.; Sun, L.; Long, F. H.; Feng, Z. C.; Ferguson, I. T. First- and second-order Raman scattering from semiinsulating 4H-SiC. Phys. Rev. B 1999, 59, 7282-7284.

[81] Kunert, H. W.; Maurice, T.; Barnas, J.; Malherbe, J.; Brink, D. J.; Prinsloo, L. Raman and photoluminescence spectroscopy from n- and p-type 6H-SIC alpha-particle irradiated. Vacuum 2005, 78, 503-508.

[82] Ramsdell, L. S. The crystal structure of $\alpha$-SiC, type 4. Am. Mineral. 1944, 29, 431-442.

[83] Lukesh, J. S.; Pauling, L. The problem of the graphite structure. Am. Mineral. 1950, 35, 125.

[84] Rohrl, J.; Hundhausen, M.; Emtsev, K. V.; Seyller, T.; Graupner, R.; Ley, L. Raman spectra of epitaxial graphene on SiC(0001). Appl. Phys. Lett. 2008, 92, 201918. 